\title{
Transitions Between Lamellar Orientations in Shear Flow
}

\author{
Ludwig Schneider, ${ }^{\dagger}$ Matthias Heck, ${ }^{\ddagger}$ Manfred Wilhelm, ${ }^{\ddagger}$ and Marcus \\ Müller*,† \\ $\dagger$ Institute for Theoretical Physics, Georg-August-Universität, Göttingen, Germany \\ $\ddagger$ Institute for Technical Chemistry and Polymer Chemistry, Karlsruhe Institute of Technology, \\ Germany \\ E-mail: mmueller@theorie.physik.uni-goettingen.de
}

\section{Abstract}

Shear flow is a versatile strategy to align microphase-separated morphologies of diblock copolymers over macroscopic scales. Details of the local mechanism of reorientation towards the steady, non-equilibrium state, however, are only incompletely understood.

Using large scale molecular simulation as well as experiments, we study the shear-alignment mechanism using lamella-forming, symmetric, unentangled diblock copolymers in steady and oscillatory shear flow. First we study homogeneously oriented systems and investigate the stability of different orientations with respect to the shear flow by the Rayleighian. Second, we investigate the process of reorienting an unstable grain with parallel orientation embedded in a matrix of a stable, perpendicularly oriented lamellae.

We observe two different reorientation mechanisms as a function of the shear rate: A fast transition, which is comparable to experimental conditions in Large Amplitude Oscillatory Shear (LAOS) tests, and a slower transition occurring at lower shear rates. We show that for high shear rates the long-range orientational order of the lamellae inside the unstable grain disintegrates, while the grain remains spatially structured with the same characteristic length scale (similar to a microemulsion). At lower shear rates, however, we observe a shrinking of the unstable grain, i.e. a directed movement of the grain boundaries. Additionally, we compare the results of steady shear with oscillatory shear.

\section{Introduction}

Diblock copolymers feature multiple interesting, tunable morphologies, which can be designed by simply accessible parameters like the volume fraction and incompatibility of the involved polymer types. ${ }^{1}$ The microphase separation makes these materials ideal candidates for the production of self-assembled nano-structures. For the majority of self-assembly applications it is of great interest to gain control over the orientation of the spatially modulated morphologies on large length scales. $^{2}$ The diblock copolymers can form three major equilibrium phases: lamellae, hexagonally packed cylinders and spheres on a body-centeredcubic lattice. ${ }^{3,4}$ In this work we focus on the dynamics of lamellar grains in chemically symmetric, AB-block copolymer systems, where the dynamics of the blocks is similar. Aligning self-assembling polymer structures by an external shear field is a known experimental method to achieve macroscopically ordered phases. ${ }^{5-15} \mathrm{~A}$ lamellar configuration is translationally invariant in two dimensions. The same is valid for a steady shear flow. As a result there are three possible orthogonal configurations of lamellae in shear flow. The orientations are sketched in Figure 1. Koppi et al. ${ }^{16}$ for example investigated poly(ethylene-propylene)- $b$ poly(ethylethylene) (PEP-b-PEE) diblock copoly- 
mer melts in oscillatory shear flow. Patel et al. ${ }^{17}$ and Riise et. al. ${ }^{18}$ investigated polystyrene- $b$ polyisoprene (PS- $b$-PI). Depending on the polymer chemistry, frequency, and temperature their results demonstrated different orientations of the lamellae to the shear flow as stable. An overview about the experimental findings of block-copolymers in shear flow can be found for example in the review of Chen and Kornfield. ${ }^{19}$
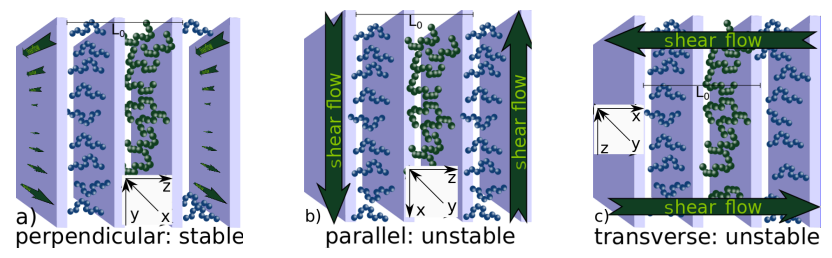

Figure 1: Lamellar configuration with respect to a steady shear flow. The stability of the orientations correspond to our findings as discussed in the following sections. a) perpendicular: the gradient of the shear flow is inside the lamellar planes - the configuration is stable in shear flow. b) parallel: the polymers in the lamellae oppose each other and slide along as in two opposing polymer brushes the configuration is unstable for the investigated system and under the applied conditions. c) transverse: the flow is perpendicular is to the planes of the lamellae, it is unstable.

In addition to theoretical considerations of block copolymers in shear flow i.e., ${ }^{20}$ several simulation studies of block-copolymers have been performed with different models and techniques. ${ }^{6,21-25}$ Liu et al. ${ }^{22}$ used a Dissipative Particle Dynamics (DPD) model and found the perpendicular orientation of the lamella to be more stable the the parallel orientation. Fraser et al. ${ }^{21}$ found the same stability with a Lennard-Jones based model of short polymers and could quantify the stability for all shear rates via the entropy production rate. Peters et al. ${ }^{6}$ compared two different simulation algorithms - Brownian dynamics and DPD molecular dynamics simulations - and found that the choice of the algorithm and shear rate has an influence on the transitions between the different orientations. All of these numerical studies investigated the stability of a homogeneous, perfectly ordered lamellar phase in the simulation boxes. Furthermore, the orientation of lamellae forming systems in shear flow is of interest for other applications. For example, Guo et. al. ${ }^{26}$ and Soddemann et. al. ${ }^{27}$ studied lamellae forming amphiphilic systems. Interestingly, they found a very similar pathway at high shear rates.

Macroscopic samples of block copolymer systems formed by self-assembly are seldom defectfree. A description of these materials is often more accurate in terms of mesoscopic regions of homogeneous orientation i.e. grains. In addition to grains and their boundaries, local defects play an important role in these systems. ${ }^{2}$ In this work we are investigating the interaction between a stable and an unstable grain and the concomitant, shear-induced transition mechanism. The local dynamics of this process is not well understood yet, ${ }^{28}$ and computer simulations can contribute to a deeper understanding.

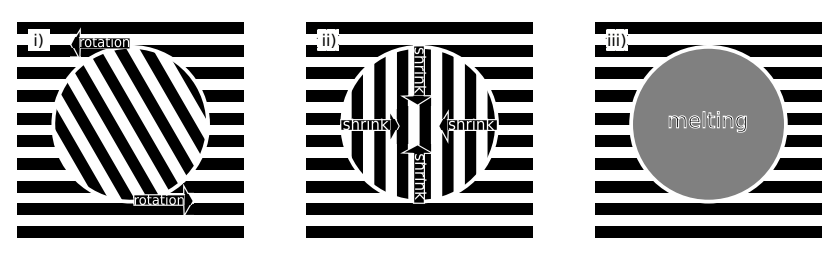

Figure 2: The hypotheses of the lamellar transitions: i) rotation of the unstable lamellar configuration in the matrix of the stable configuration. ii) shrinking of the domain or iii) melting of the unstable domain followed by a reorganization in the stable orientation. The sketch is schematic for grain sizes of about $1-10 \mu \mathrm{m}$ formed by lamellae with a natural spacing in the order of $L_{0} \approx 10-50 \mathrm{~nm}$.

For the transition of unstable grains to the stable phase three different mechanisms have been proposed. Figure 2 schematically depicts these options. The possibility i) is a rotation of the entire unstable grain inside the grain boundaries. For this transition only the global lamellar orientation inside the grain changes its direction, while the lamellar phase and the domain size remain unchanged. In this transition the molecules perform a collective mesoscopic rotational movement with the entire grain.

Hypothesis ii) describes the transition as a shrinking of the grain size, while the lamellar orientation remains unaltered. This process moves the grain boundary slowly by shrinking the unstable domain. The time to eliminate an unstable grain scales with the size of the grain, because the boundary has to cross the entire area of the grain. 
The last hypothesis iii) is the homogeneous melting of the unstable domains. The energy intake of the shear flux destroys the lamellar order inside the unstable domain. As a result the unstable domain takes an intermediate state in which the spatial modulation of the densities of the two segment densities is lost, followed by a reorientation process of the chain molecules forming a new stable domain. The new domain merges with the surrounding matrix to form a macroscopically ordered phase.

To investigate and understand the transition process, we perform Molecular Dynamics (MD) simulations with a DPD thermostat of a soft, coarsegrained model. We start our study with a quantification of the stability of the different orientations in steady shear flow via computer simulations. With the obtained stability we construct a configuration of an unstable grain embedded in a stable grain. This enables us to study different transition mechanism from the unstable grain towards the stable orientation. For low shear rates the boundary between the two grains plays an important role. We conclude our numerical studies with investigating the influence of the oscillatory shear on this mechanism.

We compare the simulation results and predictions with LAOS experiments. ${ }^{29}$ The chosen experimental model system is particularly suited because the dynamics of the two chemical blocks, reflected in the glass transition temperature $T_{g}$ and the viscosity $\eta$, is similar. The similar dynamics reduces the computational effort for the comparison.

\section{Models and Methods}

In this study we investigate both, computer simulations of individual grains and LAOS experiments of macroscopic polymer samples. We start with a description of the model used for the computer simulations. The experimental setup is described later in this section.

\section{Soft, Coarse-Grained Model for Com- puter Simulations}

For the investigation of the transition between lamellar orientations it is necessary to cover long length and time scales, because at least two grains consisting of multiple lamellae have to be simulated to capture grain-boundary effects. Depending of the re-orientation mechanism the transition from one lamellar configuration to another can take several relaxation times of the individual chain molecules. As a result the description of the polymer system with soft coarse-grained models is necessary. ${ }^{30}$

Multiple monomers are represented by an effective interaction center. Since an effective segment of our highly coarse-grained model represents a portion along the molecular backbone that comprises many statistical segments, its length is Gaussian distributed according to the central limit theorem. This Gaussian distribution of the coarse-grained segment length is modeled by harmonic bonds

$$
V_{\text {bond }}(r)=\frac{1}{2} k r^{2} \text {. }
$$

We choose the harmonic spring constant to be $k=(3+\sqrt{6}) \frac{k_{\mathrm{B}} T}{\sigma_{i}^{2}}$ and the degree of polymerization $N=128$. Both blocks $\mathrm{A}$ and B consist of $N_{A}=N_{B}=64$ monomers, making the polymer symmetric. We use the symbol $R_{e o}=$ $b_{0} \sqrt{N-1}=\sqrt{\frac{3(N-1) k_{B} T}{k}}$, the statistical equilibrium end-to-end distance as In contrast, We use the symbol $R_{\mathrm{e}}$ for the actual measured chain extension. The average end-to-end distance of the chains in a homopolymer melt is close to statistical value $R_{\mathrm{e}}^{\text {homo }}=(8.4 \pm 0.2) \sigma=(1.01 \pm 0.03) R_{e o}$. The invariant degree of polymerization $\overline{\mathcal{N}}$ characterizes a polymer melt via the number of interacting poly$\operatorname{mers} \overline{\mathcal{N}}=\left(\frac{\rho_{0} R_{e o}^{3}}{N}\right)^{2}$. We set $\sqrt{\overline{\mathcal{N}}}=32$, which avoids strong thermal fluctuations of the densities that increase with decreasing $\overline{\mathcal{N}}$. This choice implicitly defines the monomer density $\rho_{0}$. We expect our results to be transferable to higher values of $\overline{\mathcal{N}}$.

The non-bonded interaction serves two purposes in the soft coarse-grained model i) restraining density fluctuations and ii) mimicking the interaction of non-bonded particles of unlike types. We use an empiric quadratic potential as pair interaction.

$$
V_{\mathrm{m}}(\boldsymbol{r})= \begin{cases}\frac{k_{B} T}{2} v_{i j}\left(1-\frac{|\boldsymbol{r}|}{\sigma_{i}}\right)^{2}<\infty & \text { for }|\boldsymbol{r}|<\sigma_{i} \\ 0 & \text { for }|\boldsymbol{r}| \geq \sigma_{i}\end{cases}
$$


$\sigma_{i}$ describes the interaction range and defines the length scale of the reduced units for the simulations. This shape of the non-bonded pair interaction is equivalent to the pair interaction used in the DPD scheme. ${ }^{31}$ The parameter $v_{i j}$ controls the interaction strength. To control the compressibility of the polymer liquid we choose $v_{A A}=v_{B B}=5$ for the interaction between monomers of the same species, unless stated otherwise. The generic repulsion between unlike types is realized by setting $v_{A B} \approx 5.29$, which approximates a FloryHuggins parameter of $\chi N=32 .^{1}$ A repulsion of this strength results in an intermediately strong microphase separation, ${ }^{3}$ which is compatible with many experimental situations.

We consider the canonical ensemble (n,V,T), with a constant number of polymers $n$, a constant volume $V$ and a constant temperature $T$, and use a DPD thermostat. ${ }^{32}$ This thermostat has the advantage of locally conserving the momentum, because friction is added pairwise to the particle forces. This feature is crucial in our study, because we apply a shear flux to the simulation. The DPD thermostat adds a friction force $\boldsymbol{F}^{D}$ and a random force $\boldsymbol{F}^{R}$ to the conservative pairwise forces $\boldsymbol{F}^{C}$ in the system. ${ }^{32,33}$

$$
\begin{aligned}
\boldsymbol{F}_{i j} & =\boldsymbol{F}_{i j}^{C}+\boldsymbol{F}_{i j}^{R}+\boldsymbol{F}_{i j}^{D} \\
\boldsymbol{F}_{i j}^{D} & =-\gamma^{\mathrm{DPD}} w^{D}\left(\boldsymbol{r}_{i j}\right)\left(\hat{\boldsymbol{r}}_{i j} \cdot \boldsymbol{v}_{i j}\right) \hat{\boldsymbol{r}}_{i j} \\
\boldsymbol{F}_{i j}^{R} & =w^{R}\left(\boldsymbol{r}_{i j}\right) \theta \sqrt{3} \sqrt{\frac{2 \gamma^{\mathrm{DPD}} k_{B} T}{\Delta t}} \hat{\boldsymbol{r}}_{i j} \\
w^{D}(r) & =\left[w^{R}(r)\right]^{2}=\left(1-r / \sigma_{i}\right)^{2}
\end{aligned}
$$

$\theta$ is in this context a uniformly distributed random number in the interval $\theta \in[-1,1] .{ }^{33,34}$ The friction and random forces are balanced to fulfill the fluctuation-dissipation theorem. $\gamma^{\text {DPD }}$ is an input parameter, that controls the strength of this noise and friction in the simulations.

To enable the investigation of shear flow in the simulation we apply the Reverse Non-Equilibrium Molecular Dynamics Simulation (RNEMDS)

\footnotetext{
${ }^{1}$ The relation between the $\chi$ parameter and the generic repulsion $v_{A B}$ relies on matching the energy of mixing, measured in the simulation, to the Flory-Huggins expression, $\Delta E_{\text {mix }} / k_{B} T=V \rho_{0} \chi \phi(1-\phi)$. In a mixed $\left(\chi<\chi_{\text {crit. }}=2\right)$ monomer system, $N=1$, the shape of this parabola determines $\chi$ as a function of the pair potential.
}

scheme. ${ }^{35}$ RNEMDS allows simulations with a shear flow in the simulation box. The simulation box is divided into $N_{\text {slabs }}$ slabs in the direction of the desired shear gradient. In periodic boundary conditions two slabs with maximum spatial distance are selected. Between these slabs velocity components in the direction of the shear flow can be swapped to achieve a momentum flux. The result is a stress controlled simulation. The thickness of these slabs is chosen as small as possible in order to keep the disturbed regions small, but the volume of the slabs has to be sufficiently large to contain an acceptable number of particles for the swapping algorithm. In this study, we chose the slab thickness in the interval $0.12 \leq L_{\mathrm{s}} / R_{e o} \leq 0.192$. In addition, we positioned the disturbed slabs away from regions of interest i.e. grain boundaries.

The original RNEMDS scheme ${ }^{35}$ proposes a constant swapping rate of the velocity components. In contrast, we implemented an algorithm, which takes an target integrated momentum flux $J_{\text {target }}(t)=\int_{0}^{t} \mathrm{~d} t^{\prime} j_{p}^{\text {target }}\left(t^{\prime}\right)$ as an input. In each time step the deviation of the target and the actual integrated momentum flux is calculated and velocity components are swapped in order minimize this deviation. Details on this scheme can be seen in the implementation of HOOMD-blue and its documentation. ${ }^{36}$ This modified algorithm allows the simulation of more complex situations e.g. oscillatory shear.

All simulations are performed with the HOOMDblue simulation package ${ }^{37-40}$ in the version $\mathrm{v} 2.1 . \mathrm{x}$ with custom plugins.

\section{Investigations on Poly(styrene)- $b$ - poly(2-vinylpyridine) as a Model Sys- tem}

The local polymer dynamics correlate with the glass transition temperature of the polymer. We choose a diblock copolymer model system with a similar glass transition temperature $\left(\Delta T_{g} \approx 14 \mathrm{~K}\right)$ in the different polymer blocks for the comparison between computer simulations and rheological experiments. ${ }^{29}$ Furthermore, the molecular weight distribution of the polymer system has to be as narrow as possible to better match the simulation models. ${ }^{41}$ Reactions in anionic polymerization lead- 
ing to termination or undefined branching of the propagating polymer chain are in principle completely absent, in contrast to other polymerization methods. Thus this technique is well suited for the synthesis of well defined model systems. ${ }^{42-44}$ With this technique linear diblock copolymers are synthesized with a low dispersity index $(\mathrm{PDI} \leq 1.2)$ and consequently an exquisite control over molecular weight. For this reason and to be able to examine the sample by small angle X-ray scattering (SAXS), the diblock copolymer poly(styrene)- $b$ poly(2-vinylpyridine) (PS- $b$-P2VP) was chosen as a model system. This block copolymer shows a strong microphase separation ${ }^{45-47}$ and the difference in electron density in the two polymer blocks is sufficiently high to investigate the morphology via SAXS. ${ }^{29}$ We describe in the subsequent section the orientation behavior of a PS- $b$-P2VP sample. The molecular weight of the polystyrene block is $8400 \mathrm{~g} \mathrm{~mol}^{-1}$ and the molecular weight of the poly-2-vinylpyridine block is $8600 \mathrm{~g} \mathrm{~mol}^{-1}$. The sample name is abbreviated as $\mathrm{PS}(8.4) \mathrm{P} 2 \mathrm{VP}(8.6)$ in the following. This molecular weight enables to erase orientation of the lamellae (e.g. during sample preparation) by heating the polymer above the order-disorder transition temperature $T_{O D T}$ as described later. At this temperature $\chi N$ is so low $\left(\chi N<10.5^{48}\right)$ that the two diblock copolymer melt becomes homogeneous.

Synthesis of the Model System The diblock copolymer was synthesized using anionic polymerization. Thus high vacuum techniques were used as described in more detail in the literature. ${ }^{44}$ All glassware was heated to approximately $600{ }^{\circ} \mathrm{C}$ and flushed with argon in order to remove all traces of adsorbed water. Reagents were added from vessels directly connected to the reaction flask or under argon counterflow.

Solvents Tetrahydrofurane (THF) $(\geq 99.5 \%$, Carl Roth) was refluxed over calcium hydride for several days. It was further purified with sodium and benzophenone where a purple color indicated that all traces of water were removed from the solvent. The storage flask was directly connected to the vacuum line and thus the solvent can be removed without contamination. Just before use, the THF was distilled into the reaction flask. Toluene was stirred over calcium hydride for sev- eral days and then further purified and stored over 1,1-Diphenylethylene and n-Buthyllithium.

Reagents and Monomers Secondary butyllithium (sec-BuLi) (1.4 mol $\mathrm{l}^{-1}$ in hexane, Sigma Aldrich) was used as received. 1,1Diphenylethylene (DPE, $98 \%$, Alfa Aesar) was purified by the addition of n-Butyllithium until a dark red colour persisted. Diphenylethylene was distilled under high vacuum at $85^{\circ} \mathrm{C}$. Subsequently THF was added to achieve a concentration suitable for the sythesis of the polymer samples. Methanol ( $\geq 98.5 \%$, VWR Chemicals), which was used for the termination of the polymerization reactions, was frozen with liquid nitrogen and subsequently thawed to remove oxygen. This was necessary to prevent coupling of two macroanions leading to a doubling of the molecular weight. This procedure was repeated until no more gas bubbles were observed.

2-Vinylpyridine (97\%, Acros Organics) was stirred over calcium hydride (1 $\mathrm{g}$ for $50 \mathrm{ml})$ overnight. In order to remove all remaining traces of water, the monomer was distilled into another Schlenk flask containing $\mathrm{Et}_{3} \mathrm{Al}\left(1.0 \mathrm{~mol} \mathrm{l}^{-1}\right.$ in hexane, $1 \mathrm{ml} \mathrm{Et}_{3} \mathrm{Al}$ solution for $20 \mathrm{ml} 2 \mathrm{VP}$ ). The hexane was removed under vacuum prior to the $2 \mathrm{VP}$ distillation. The 2-vinylpyridine was stirred for 1 hour and then distilled at $95{ }^{\circ} \mathrm{C}$ under vacuum. It was used directly or stored in an ampule under argon at $-18{ }^{\circ} \mathrm{C}$ for $\leq 5$ days.

Styrene (99.5\%, Carl Roth) was stirred over calcium hydride ( $1 \mathrm{~g}$ for $50 \mathrm{ml}$ ) over night. Then it was distilled into another Schlenk flask containing dibutyl magnesium (1.0 mol $\mathrm{l}^{-1}$ in hexane, $1 \mathrm{ml}$ dibutyl magnesium solution for $20 \mathrm{ml}$ styrene). The hexane was removed under vacuum prior to distillation. The styrene was stirred for 3 hours over the dibutyl magnesium and then was distilled into an ampule under vacuum at $90-95^{\circ} \mathrm{C}$. The styrene in the ampule was either used directly or was stored under argon at $-18{ }^{\circ} \mathrm{C}$ for up to two weeks.

\section{Synthesis of PS- $b$-P2VP}

The PS block was synthesized in toluene at ambient temperatures inside an ampule. This was done to keep the PDI of the polystyrene block as low as possible. In order to reduce the reactivity of the macroanion, DPE was added and the reaction mixture was stirred for $2 \mathrm{~h}$. Prior to the synthesis of the P2VP-block, THF was distilled 
into a reaction flask or added from an ampule (9:1 THF/Toluene, e.g. $100 \mathrm{ml}$ for $7 \mathrm{~g}$ PS- $b$-P2VP). The polystyrene solution was added to the THF and cooled to $-50{ }^{\circ} \mathrm{C}$. Subsequently the $2 \mathrm{VP}$ was added under argon counter flow. The reaction mixture was stirred for $1 \mathrm{~h}$ at $-50{ }^{\circ} \mathrm{C}$ and then stirred for $24 \mathrm{~h}$ at room temperature. The reaction was terminated by the addition of degassed methanol. The product was precipitated in cold $\left(T \approx-30^{\circ} \mathrm{C}\right)$ low boiling petroleum ether and dried under vacuum.

Molecular weight determination The molecular weight of the polymer samples was determined via gel permeation chromatography (GPC) and ${ }^{1} \mathrm{H}$ NMR. The GPC equipment was from Polymer Standard Service, (Mainz, Germany) specifically the Agilent 1200 series. Two PSS SDV Lux $8 \mathrm{~mm}$ x $30 \mathrm{~mm}$ columns with a pore size of $10^{3}$ respectively $10^{5} \AA$ were used. The solvent was THF at $25{ }^{\circ} \mathrm{C}$ with a flow rate of $1 \mathrm{ml} \mathrm{min}{ }^{-1}$. The total number average molecular weight $M_{n}$ was determined using ${ }^{1} \mathrm{H}$ NMR. The samples were dissolved in deuterated chloroform $\left(\mathrm{CDCl}_{3}, 99.8 \%\right.$, Sigma Aldrich) for the NMR experiments. A Bruker Avance 111 Microbay $400 \mathrm{MHz}$ spectrometer was used and typically measured 128 scans. The polydispersity index of the blockcopolymer was determined using GPC.

Differential Scanning Calorimetry Measurements The glass transition temperature was determined using a Mettler Toledo DSC30 differential scanning calorimeter. The temperature ramp rate was $10 \mathrm{~K} \mathrm{~min}^{-1}$. All polymer samples were dried for several days at $70{ }^{\circ} \mathrm{C}$ under vacuum before measurements.

\section{Varying Block Dynamics in the Computer Sim-} ulations In the experimental situation the mobility of the monomers of the A- and B-block are not identical. It is possible to achieve similar block dynamics via an appropriate choice of the monomers, ${ }^{29}$ but differences are inevitable. The difference in the glass transition temperature $T_{g}$, which reflects the polymer dynamics for example is $\Delta T_{g} \approx 14 K$ for the system polystyrene- $b$-poly2 -vinylpyridine, which is used in this study. As a consequence, we mimic this characteristic in our simulations.

In our soft, coarse-grained model the block mobility is determined by the friction. The friction is, as a result of the soft model, not dominated by the pair potential, but by the thermostat. In the DPD scheme the parameter $\gamma^{\text {DPD }}$ (Equation 3) determines the strength of the thermostat. This parameter controls the amplitude of the drag and random forces applied to the particle pairs. To achieve distinct block dynamics we tune $\gamma^{\text {DPD }}$ as a function of the involved particle types. Inside the A-block we employ a lower friction, thus higher mobility, than in the B-block $\gamma_{A A}^{\mathrm{DPD}}<\gamma_{B B}^{\mathrm{DPD}}$. For interactions of different particle types the average is used $\gamma_{A B}^{\mathrm{DPD}}=\frac{1}{2}\left(\gamma_{A A}^{\mathrm{DPD}}+\gamma_{B B}^{\mathrm{DPD}}\right)$.

From the long time regime of Mean Squared Displacement (MSD) we are able to determine the diffusion constant $D$ for the homopolymers of a specific type. ${ }^{49}$

$$
\lim _{t \rightarrow \infty}\left\langle\left(\boldsymbol{r}_{i}(0)-\boldsymbol{r}_{i}(t)\right)^{2}\right\rangle=6 D t
$$

The diffusion constant allows the definition of a relaxation time for the polymers $T_{R}=\frac{R_{e o}^{2}}{D}$.

For the A type polymer we use $\gamma_{A A}^{\mathrm{DPD}}=0.5$, which results in a relaxation time of $T_{R}^{A}=$ $(10200 \pm 100) \tau$. With a B type mobility of $\gamma_{B B}^{\text {DPD }}=1.5$ and $T_{R}^{B}=(13820 \pm 80) \tau$ the ratio between the slow and the fast polymer block is about $T_{R}^{B} / T_{R}^{A} \approx 1.35$. $\tau$ is the internal reduced time unit of the simulations for the specific monomer combination used in this study. This situation is achievable in experimental setups (Poly(styrene)- $b$ poly(2-vinylpyridine) as reported in Ref. ${ }^{29}$

\section{Orientation and SAXS Experiments}

Preparation of the Samples for Rheological Experiments A PW-H HKP300 press from $\mathrm{P} / \mathrm{O} /$ Weber (Remshalden, Germany) was used for the sample preparation. The dried polymer powder was placed in between two teflon disks inside a mold. The amount of polymer was chosen to form disks of $1 \mathrm{~mm}$ thickness (e.g. $\approx 130 \mathrm{mg}$ per sample with a diameter $\varnothing=13 \mathrm{~mm})$. Vacuum was applied to the mold after it was placed in the press and the sample was heated to $180{ }^{\circ} \mathrm{C}$. Pressure ( $p \approx 2 \mathrm{kN} \mathrm{cm}^{-2}$ ) was applied to the piston to form the disk shape and remove any trapped air. The sample was removed from the press at ambient temperature. All measured samples were checked visually for the absence of trapped air or cracks. 
Rheological Determination of the OrderDisorder Transition Temperature $T_{O D T}$ An Advanced Rheometric Expansion System (ARES) from TA Instruments (Newcastle, USA) was used in oscillatory mode for these investigations. Temperature sweep experiments were conducted using homemade plate-plate geometries with a diameter of $25 \mathrm{~mm}$ made from Invar. To measure $T_{O D T}$ a temperature-sweep experiment was performed over a large temperature range to find the temperature, at which there was a sudden decrease in the storage modulus $G^{\prime}(T)$ and loss modulus $G^{\prime \prime}(T)$. Subsequent experiments over a narrower temperature range at low heating and cooling rates $\left(1 \mathrm{~K} \mathrm{~min}^{-1}\right)$ were conducted to confirm and further determine the transition temperature more precisely. The $T_{O D T}$ was determined as shown in Figure 3 . The experiments were performed using an angular frequency of $\omega_{1}=2 \pi \mathrm{rad} \mathrm{s}^{-1}$ and a shear strain amplitude of $\gamma_{0}=0.01$. All experiments were conducted under a nitrogen atmosphere to prevent oxidative degradation of the polymers. The heating and cooling procedure was repeated several times to ensure that the change in $G^{\prime}(T)$ and $G^{\prime \prime}(T)$ originated from the order-disorder transition and not from thermal decomposition. The polymer samples were analyzed by GPC before and after the temperature-sweep experiments and no change in either $M_{w}$ or a broadening of the molecular weight distribution due to chain scission was observed within experimental reproducibility.

Orientation Experiments In order to remove the pre-orientation of the lamellae, the sample $\mathrm{PS}(8.4) \mathrm{P} 2 \mathrm{VP}(8.6)$ was heated $5^{\circ} \mathrm{C}$ above $T_{O D T}$ for approximately 5 minutes and then cooled to the temperature, at which the orientation experiment was conducted. Large amplitude oscillatory shear was used to align the lamellae. The experimental parameters for the orientation experiment over 2000 s, which is illustrated in Figure 6 were temperature $T=200{ }^{\circ} \mathrm{C}$, strain $\gamma_{0}=1.5$, frequency $\omega_{1} / 2 \pi=1 \mathrm{~Hz}$. The sample was cooled to room temperature freezing the orientation of the aligned lamellae. For spatially resolved small angle X-ray scattering (SAXS) experiments: a stripe was cut from the polymer disk, which was further divided for the scattering experiments. The dimensions of the samples were checked with a digital cantilever

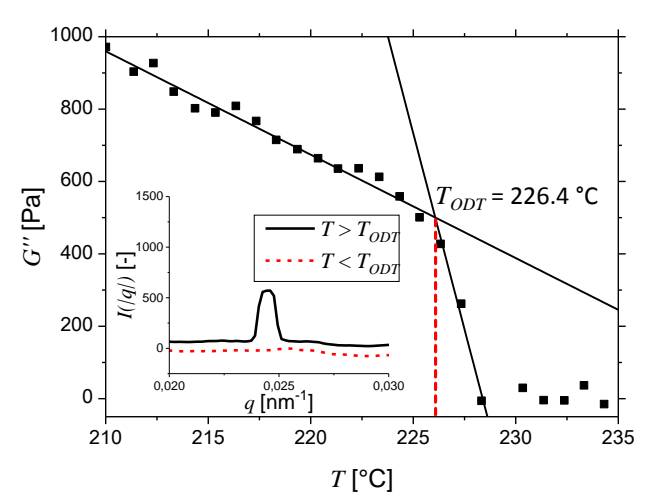

Figure 3: Determination of $T_{O D T}$, which is defined as the intersection between linear fits to the data over the temperature range $210{ }^{\circ} \mathrm{C}$ to 220 ${ }^{\circ} \mathrm{C}$ and the data at the sharp decrease of $G$ " (three data points). The sample $\mathrm{PS}(8.4) \mathrm{P} 2 \mathrm{VP}(8.6)$ was measured at $\omega_{1}=2 \pi \mathrm{rad} \mathrm{s}^{-1}, \gamma_{0}=0.01, \Delta T$ $=1{ }^{\circ} \mathrm{C} \mathrm{min}{ }^{-1}$. The $T_{O D T}$ was determined to be $226.4{ }^{\circ} \mathrm{C}$. The inset shows the scattering Intensity $I(|q|)$ of the phase separated polymer melt (normal line, $T=220^{\circ} \mathrm{C}$ ) and the homogeneous melt (dotted line, $T=230{ }^{\circ} \mathrm{C}$ ). The data was shifted for a better readability.

enabling the calculation of the measurement position on the original polymer disk.

SAXS Ex-situ SAXS measurements were conducted on a Hecus S3-Micro X-ray system with a point microfocus source, 2D-X-Ray mirrors and a two dimensional CCD-Detector from Photonic Science.

In-situ Rheo-SAXS measurements were conducted at the HASYLAB (DESY, Hamburg, Germany), at a wavelength of $0.154 \mathrm{~nm}$. The used rheometer was a Mars II (Thermo Scientific). A custom made Vespel parallel plate geometry with a diameter of $36 \mathrm{~mm}$ containing thin windows of $0.3 \mathrm{~mm}$ thickness at $14 \mathrm{~mm}$ distance from the center of the plate $\left(\gamma_{\text {local }}=0.778 \cdot \gamma_{0}\right)$ were used for the orientation experiments. The scattered intensities were recorded by a 2D detector (Pilatus 300k). A similar setup is described in the literature in more detail. ${ }^{15,50}$ 


\section{Results and Discussion}

An investigation of the transition between different orientations of the lamellae in shear flow requires the knowledge which configurations are stable and which configurations are meta- or unstable in shear flow. We begin with a stability analysis of the nonequilibrium steady state and conclude the investigation with an analysis of two transition between an unstable and a stable lamellar configuration.

\section{Stability of the Lamellar Orientations}

We introduced the three orthogonal options of the lamellar orientation with respect to the shear flow in Figure 1. Now we are discussing the stability of these orientations via computer simulations.

As a first step to determine the stable lamellar orientation we can eliminate the transverse configuration, which corresponds to Figure $1 \mathrm{c}$ ). The flow is deforming and stretching the lamellae, until the lamellae rupture, so this configuration is a priori unstable. For the other two orientations there exists no such geometric argument to determine their stability. So we employ the concept of the Rayleighian to determine their stability, which has been suggested as the free-energy equivalent for non-equilibrium situations. ${ }^{51,52}$ Our approach to employ the Rayleighian in steady-state situations is similar to the approach of Fraser et al. ${ }^{21}$ The latter authors used the entropy production and rate of energy dissipation as a measure of stability in non-equilibrium situations.

Computer Simulations We simulate both orientations in a box of dimensions $2 L_{x}=2 L_{y}=L_{z}$ with the shear gradient in the Z-direction. The normal vectors of the lamellar interfaces are pointing in the Y-direction for the perpendicular configuration (Figure $1 \mathrm{a}$ ) and in the $\mathrm{Z}$-direction for the parallel configuration (Figure $1 \mathrm{~b}$ ). The box dimensions, $L_{x}=2 L_{0}$, are chosen to be commensurate with natural lamellar spacing, $L_{0}=1.74 R_{e o}$, for the chosen incompatibility, $\chi N=32$. Only for this stability analysis, we use a lower compressibility by setting $v_{A A}=v_{B B}=0.5$ and $v_{A B}=0.69$, the same friction coefficients for the components, $\mathrm{A}$ and $\mathrm{B}$, as well as the Lowe-Anderson thermostat $^{53}$ instead of the DPD thermostat. Both imple- ment the same physical concept so the results are transferable, but we use the same friction for both components $\mathrm{A}$ and $\mathrm{B}$ in this case.

The RNEMDS of homopolymers and the perpendicular configuration (see Figure 1 a) can be conducted for momentum flux, $j_{p}$. For the parallel configuration (cf. Figure $1 \mathrm{~b}$ ), however, a steady state could only be established for low shear rates. Starting with stresses of about $j_{p} R_{e}^{3} / k_{B} T \approx 65$ the lamellar orientation gradually changes. These simulations cannot be considered as steady state, so we exclude them from the stability analysis. In the remainder of this section we focus only on the steady states. The occurrence of this reorientation is already a hint that the parallel configuration might be unstable at high stresses. These gradual transition shall not be confused with the separation between the transition pathways mentioned later in this section.

The Rayleighian measured in the systems can quantify, which of two states is more stable. The Rayleighian, $\tilde{R}$, as a function strain rate, $\dot{\gamma}$, is composed of the two contributions: ${ }^{52}$

$$
\tilde{R}(\dot{\gamma})=\underbrace{\dot{F}}_{\text {change in free energy }}+\underbrace{\frac{1}{2} \Sigma T(\dot{\gamma})}_{\text {dissipated energy/time }}
$$

where the dissipated energy per unit time can be measured as the hydrodynamic quantity ${ }^{54}$

$$
\Sigma T=\frac{\eta}{2} \int_{V}\left(\frac{\partial v_{i}}{\partial x_{k}}+\frac{\partial v_{k}}{\partial x_{i}}\right)^{2}=A \int_{L_{z}} \mathrm{~d} z j_{p}(z) \dot{\gamma}
$$

The second equality, with $A$ being the area of the $\mathrm{XY}$ cross section, is only valid in the special case of momentum transport along the Z-axis.

In our simulation, however, we do not control the strain rate, $\dot{\gamma}$, but rather the stress. In order to obtain the stress-controlled Rayleighian, $R\left(j_{p}\right)$, as a function of the momentum flux, $j_{p}$, we employ a Legendre transformation, resulting in

$$
R\left(j_{p}\right)=\dot{F}-\frac{1}{2} \Sigma T\left(j_{p}\right)
$$

The steady-state condition implies that the free energy does not change, $\dot{F}=0$. Thus, minimizing the Rayleighian corresponds to maximizing the dis- 
sipation rate at controlled stress or minimizing the dissipation rate at controlled strain rate, respectively.

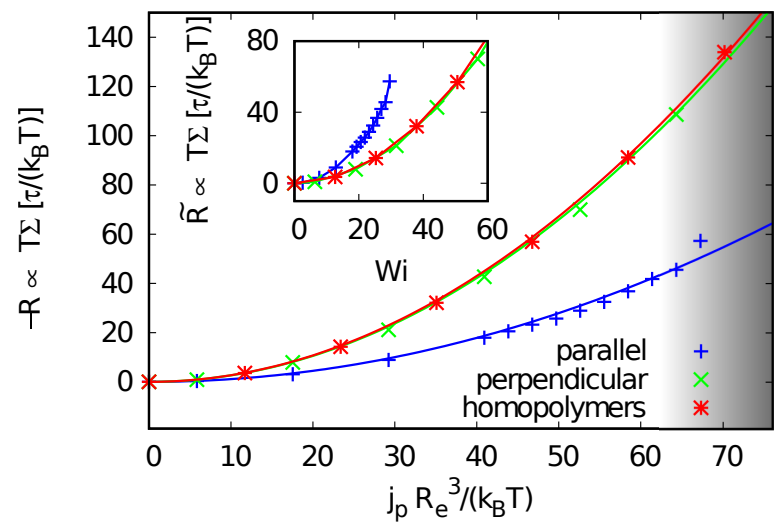

Figure 4: Dissipated energy per unit time as a function of the induced momentum flux $j_{p}$ (shear stress). The points present the simulation results whereas the lines are quadratic fits to $R \propto j_{p}^{2}$. At high stresses (gray) the unstable parallel configuration (Figure $1 \mathrm{~b}$ ) gradually changes their orientation and cannot be considered as steady state. The inset presents the same data as a function of the Weissenberg number, Wi, quantifying the shear rate.

Figure 4 plots the obtained data for steady-state simulations of the parallel and perpendicular lamellar orientation in shear flow. In the main panel, the dissipation rate, $\Sigma T=-R\left(j_{p}\right)$, is shown as a function of momentum flux (or stress), whereas the inset displays the same data, $\Sigma T=\tilde{R}(\dot{\gamma})$, as a function of the Weissenberg number, $\mathrm{Wi}=\dot{\gamma} \cdot T_{R}$. The Rayleighian of both orientations has the same trivial value at zero momentum flux or shear rate, which is expected, because both orientations represent the equilibrium state. As soon as the symmetry is broken by the shear flow, the stress-controlled Rayleighian, $R=-T \Sigma$, of the parallel configuration grows faster than for the other orientation. Since the viscosities of the parallel and perpendicular state differ, $\eta_{\|}>\eta_{\perp}$, so does the order of the dissipation rate at controlled stress or fixed strain rate, $\Sigma T=\frac{V}{2} \frac{j_{p}^{2}}{\eta}$ or $\Sigma T=\frac{V}{2} \eta \dot{\gamma}^{2}$, respectively. The quadratic dependency of the Rayleighian on $j_{P}$ or $\dot{\gamma}$, respectively, is expected - the Rayleighian remains invariant under mirroring the flow. So in the limit of small shear rates the quadratic term is the lowest non-vanishing contribution.

Figure 4 reveals that both, the minimization of
$R\left(j_{p}\right)$ at fixed stress (main panel) or the minimization of $\tilde{R}(\dot{\gamma})$ at fixed strain rate (inset), consistently indicate that the parallel configuration is less stable than the perpendicular configuration at all shear rates. The expectation is that for an infinite observation time the parallel configuration undergoes a transition to the perpendicular orientation.

The data for the homopolymer case overlaps with the obtained rate of dissipated energy of the perpendicular orientation. This indicates that the microphase separation does not alter the energy dissipation rate of the melt, if the orientation of the lamellae is stable i.e. perpendicular.

Because we are able to establish a steady state for low shear rates for the parallel configuration, we assume the unstable state is metastable. But this meta-stability seems to vanish or the transition barrier shrinks significantly for higher shear rates as it is not possible to establish a steady state anymore. The following investigations of the transition at high shear rates supports this conclusion.

To understand the difference in stability of the two orientations, we investigate the velocity profiles induced by the stress-controlled shear. In the parallel configuration the gradient of the velocity profiles crosses the interfaces of the lamellae, but not in the other two cases of homopolymers and the perpendicular orientation. Figure 5 plots the velocity profile in the simulation box for all three cases. To highlight the crossing of interfaces, in the case of the parallel configuration, the background is colored according to the local composition.

For homopolymers the velocity profile in the perpendicular lamellar orientation is linear indicating the homogeneity of the phase in the direction of the velocity gradient. For the parallel configuration the profile changes as a function of $z$. Inside half a lamella the profile shows the same gradient as in the homogeneous cases, but at the interface the slope differs. In the stress-controlled simulations the spatial stress is constant in the parallel as well. We conclude that a locally defined shear viscosity $\eta=\sigma_{s t} / \dot{\gamma}$ significantly increases at the interface, making the global configuration over all more viscous. An increased averaged viscosity increases the total energy dissipation (Equation 6), so consequently the configuration is less stable.

The parallel lamellar orientation is comparable to two grafted, polymer brushes in opposition sliding 


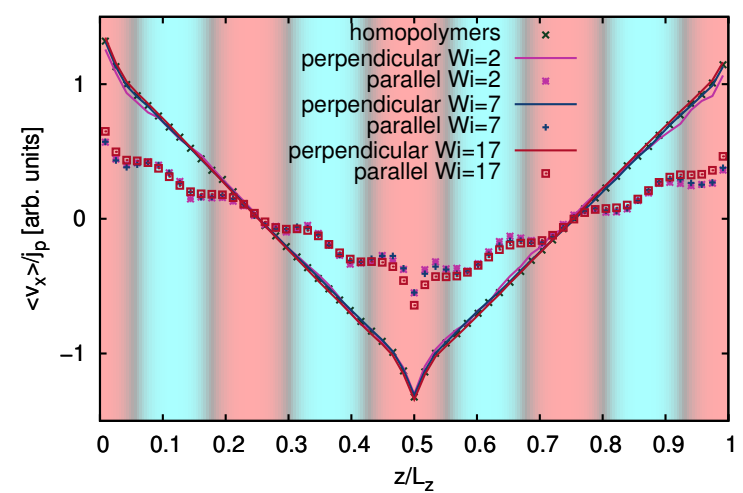

Figure 5: Spatially averaged velocity profiles of the two lamellar orientations and different shear rates (all steady states). The collapse of the normalized curves highlight the linear response of the profiles to the applied stress. In the background the color is chosen with respect to the $\mathrm{AB}$ composition of the parallel configuration. A-rich domains are colored red, B is colored in cyan, interfaces are dark. For the other two cases a corresponding background would be homogeneous. The momentum-transfer regions caused by the RNEMDS scheme are located around $z / L_{z}=0.5$ and $z / L_{z}=0$ and are not considered for the analysis.

past each other. In simulations of these systems, an explanation for the flow inversion close to the interface has been found. ${ }^{24,55,56}$ The polymers exhibit a circular motion, with a reversed movement close to the grafting surface or interface. The reverse motion slows the momentum transport close to the interfaces.

\section{Experimental Validation of the Perpendicular Orientation as Stable}

The sample PS(8.4)P2VP(8.6) oriented in the RheoSAXS setup showed an increasing scattering intensity $I$ when the lamellae were observed in the normal direction as an increasing part of the lamellae is oriented perpendicularly (see Figure 19 on page 18). Additional ex-situ SAXS experiments of the sample oriented as described in a later section showed X-ray scattering only in the normal and tangential direction (see inset of Figure 19 on page 18). This confirms the perpendicular orientation of the lamellae. The time evolution of the orientation was monitored by the develop- ment of the scattering intensity integrated along the angle $\theta$ (see Figure 6 inset i)). With an increase of $I$ the standard deviation of a Gaussian fit to the intensity maxima decreases. The perpendicular orientation was additionally confirmed in dielectric experiments using a rheo-dielectric combination that enables the in-situ monitoring of orientation processes in block copolymers. A detailed description of this technique applied to block copolymers can be found in the literature. ${ }^{57}$ As the lamellae are oriented perpendicular to the electrodes the dielectric loss $\epsilon^{\prime \prime}(t)$ increases until a plateau is reached (see Figure 7). The orientation also depends on the polymers the diblock copolymer consist of. As an example the lamellae of the diblock copolymer poly(ethylene/propylene)$b$-poly(ethylethylene) were reported to orient in a perpendicular orientation at high shear rates, while the lamellae orient parallel for low shear rates. ${ }^{16}$ However, the lamellae of the diblock copolymer polystyrene- $b$-polyisoprene (PS- $b$-PI) were reported to orient parallel for high shear rates and parallel at low shear rates. ${ }^{58,59}$ These experimental findings differ from the ones for non entangled diblock copolymer with similar dynamics in both polymer blocks in this study and the findings in computer simulations. However, it has to be considered that additional factors such as the molecular weight (and thus entanglements), a different $\chi N$ and big differences in the dynamics of the different polymer blocks in PS- $b$-PI may influence the stability of the different orientations. Moreover, the differences in the block dynamics might have an effect, which leads to a different stability of the orientations. We investigated small differences in molecular dynamics and could not find a change in the stability of the orientations. ${ }^{29}$ The different preferences of the blocks for the sample holder surface always promote the parallel configuration and might explain the observed differences.

With these experiments we already started to investigate the time evolution of a melt of randomly orientated grains towards macroscopic homogeneity. In the next step we investigate the transitions via computer simulations. 


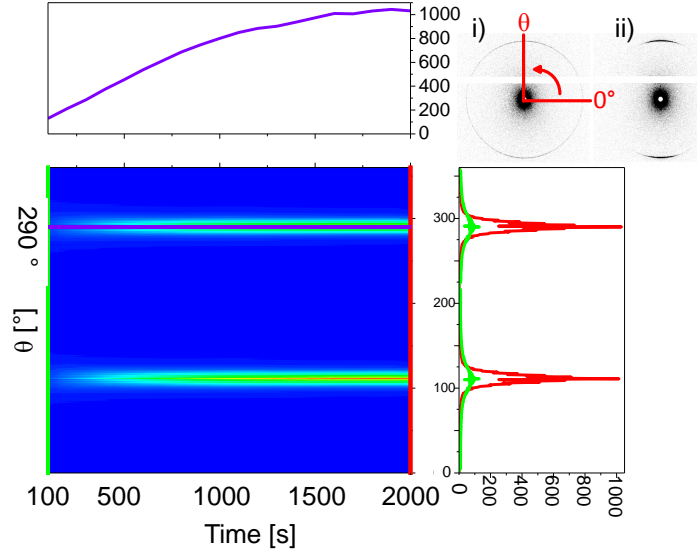

Figure 6: Time evolution of the scattering intensity $I$ during the orientation process of the lamellae of PS(8.4)P2 VP(8.6) through large amplitude oscillatory shear. $\mathrm{T}=200{ }^{\circ} \mathrm{C}, \omega_{1} / 2 \pi=1 \mathrm{~Hz}, \gamma_{0}=1.5$ $\left(\gamma_{\text {local }}=1.16\right), \mathrm{t}=2000 \mathrm{~s}$. The diffractograms in the top right show i) the state at the beginning and ii) at the end of the experiment. The data is averaged over $100 \mathrm{~s}$ to reduce noise and effects related to the oscillation and sampling rate. The angle $\theta$ is indicated in i). The diffractogramm was integrated from $\theta=0{ }^{\circ}$ to $\theta=360^{\circ}$. The scattering intensity $I$ at the angle $\theta=290^{\circ}$ (purple line) at one of the maxima is shown at the top of the graph. $I$ at the beginning (green) and at the end (red) is shown in the right graph.

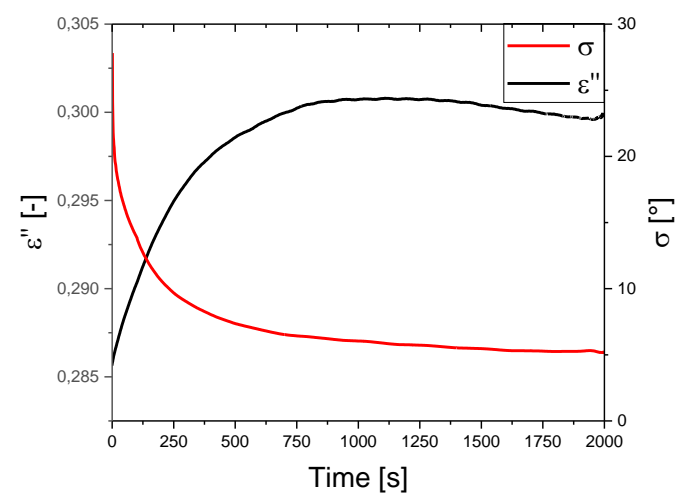

Figure 7: The time evolution of $\epsilon^{\prime \prime}(t)$ and $\sigma$ reflects the orientation process into the stable perpendicular configuration. The dielectric loss $\epsilon^{\prime \prime}(t)$ increases as the lamellae align into the perpendicular orientation. This is correlated with a decrease of the standard deviation of Gaussian fits $\sigma$ to the maximum of $I$ in SAXS experiments of $\mathrm{PS}(8.4) \mathrm{P} 2 \mathrm{VP}(8.6)\left(T=200{ }^{\circ} \mathrm{C}, \omega_{1} / 2 \pi=1 \mathrm{~Hz}\right.$, $\gamma_{0}=1.5, \mathrm{t}=2000 \mathrm{~s}, \omega_{\text {dielectric }} / 2 \pi=10^{6} \mathrm{~Hz}$ ) (refer to Figure 6). The slight decrease of $\epsilon^{\prime \prime}(t)$ is related to a minor temperature drift during the experiment.

\section{Transition from the Parallel to the Per- pendicular Configuration}

In the previous section we could show that the parallel configuration is not stable in nonlinear steady (simulation) and oscillatory (experiment) shear flow. Now we focus on the transition between the unstable parallel configuration and the perpendicular configuration at high shear rates. To investigate this transition we study a system, which is large enough to contain grains of both orientations plus two boundaries between them in the simulation box. Including the boundaries is important, because in an experimental situation we expect the samples $(10 \mathrm{~mm}$ in diameter: $\approx 1 \mathrm{~mm}$ height) to be composed of multiple grains of different orientation. The grain size is in the range of $500 \mathrm{~nm}-5 \mu \mathrm{m} .{ }^{61}$ So in the transformation of a grain into another, the boundary might play a major role, especially for the shrinking hypothesis of ii) in Figure 2.

We choose the system dimensions as $\frac{14}{3} L_{x}=$ $2 L_{y}=L_{z}=14 L_{0}$. The $\mathrm{X}$-dimension is chosen to ensure that even the shear-flow-extended chains do not interact with their periodic image. The other 


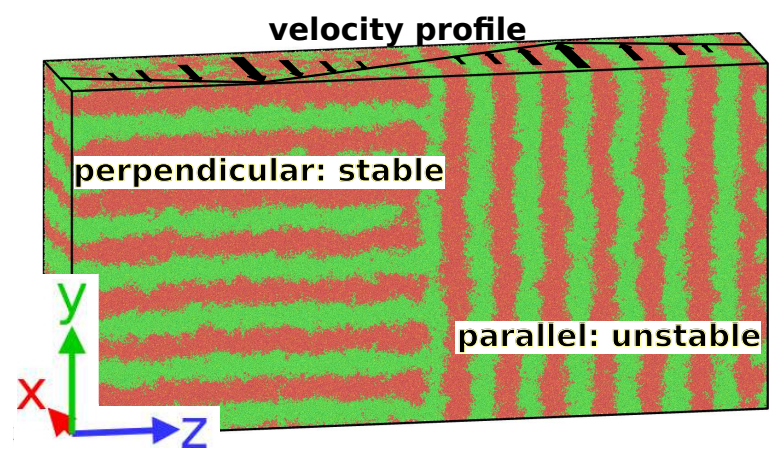

Figure 8: Snapshot of the initial configuration for the investigation of the re-orientation mechanisms. The gradient of the velocity profile is parallel to the Z-direction and the flow is parallel to the $\mathrm{X}$ direction. As a result the left-hand side of the configuration is stable (Figure $1 \mathrm{a}$ ) and the right-hand side is unstable (Figure $1 \mathrm{~b}$ ). With the periodic boundary conditions two boundaries between the grains are simulated. The direction of the shear flow is along the $\mathrm{X}$-axis - in and out of the image plane. The graphic has been created using ovito. ${ }^{60}$

two dimensions enable seven full lamellae for each grain, which is sufficient to distinguish between grain boundary and grain interior. Figure 8 presents an image of the initial setup. The momentumtransfer regions of the RNEMDS scheme are set up in the center of each grain to avoid any undesired influences on the boundary dynamics.

\section{Description of the Fast Transition at High Shear Rates: Microemulsion-like Pathway}

The investigation is intended to study the simplest case of a transition in a steady shear flow, but the momentum flux $j_{p}$ makes the parallel region unstable, as demonstrated in a previous section. Consequently, we have to make sure that during the shear start-up from the equilibrium configuration to the steady-shear flow the transition does not start already. As an indication for the shear start up we investigate the velocity profile in the configuration.

The establishment of a steady shear profile (Figure 9) can be achieved after a fraction of the chain relaxation time $T_{R}$. A linear profile, in the case of the perpendicular configuration, can be established. For the parallel part the already discussed wavelike profile is observed. During this time period no change of the spatial composition is detectable.

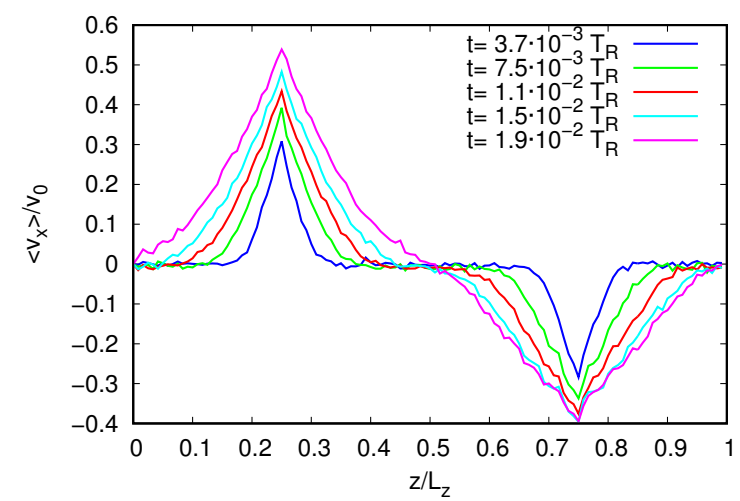

Figure 9: Time evolution of the velocity profiles during the shear start-up phase. The propagation of the velocity profile is a fast process, so after only about $0.02 T_{R}$ a steady flow is established.

It can be assumed that the shear start-up does not initiate and influence the transition itself. In combination with the previously defined relaxation time $T_{R}$ the steady shear profile enables the definition of two Weissenberg numbers $\mathrm{Wi}=\dot{\gamma} \cdot T_{R}$ in our stresscontrolled simulations. The average slope of the velocity profile $\dot{\gamma}_{\perp}$ of the perpendicular configuration is different compared to the parallel part of the simulation box. The resulting Weissenberg numbers are $\mathrm{Wi}_{\perp} \approx 200$ and $\mathrm{Wi}_{\|} \approx 94$. This shear rate is well above the steady-state shear rates applied for the stability analysis, compare with Figure 4. We show in the following, that at this high shear rate the transition from parallel to perpendicular happens fast. In a following section we also investigate the mechanism of the transition from a metastable state i.e. small shear rate.

The next phase of the transition is a process, that we classify as a disintegration of the orientational order of parallel lamellae. But this disintegration only affects the anisotropy of the lamellae, but not their local segregation. Figure 10 shows a snapshot of the configuration at the beginning of the transition. Clearly, in the right hand of the simulation box the lamellar orientation of the parallel grain is disturbed, but domains with a characteristic size are still present. The left half of the simulation box containing the perpendicular morphology is not altered by the applied shear flow.

For further investigations of this intermediate state of the transition we analyze the orientational order to gain insights about the structure. The sys- 


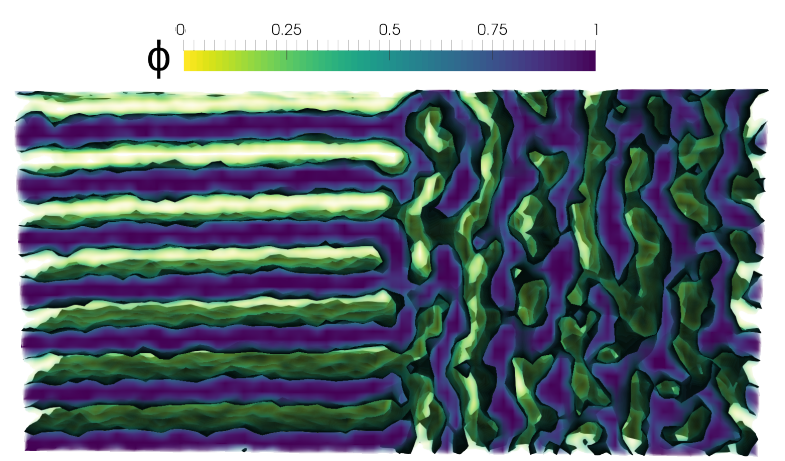

Figure 10: Snapshot of the order parameter $\phi(\boldsymbol{r})=$ $\frac{\rho_{A}(\boldsymbol{r})}{\rho_{A}(\boldsymbol{r})+\rho_{B}(\boldsymbol{r})}$ of the disintegration of the parallel configuration. Isosurfaces $\phi=0.5$ are drawn in blue black. The snapshot has been taken at $t \approx 0.18 T_{R}$ after application of the shear flow. The right half of the simulation undergoes a transition from the parallel to the perpendicular orientation. The melt is still phase separated, but the lamellar orientation is disturbed. The direction of the shear flow is as in Figure 8. The graphic has been made using paraview. ${ }^{62}$

tem symmetry allows us to average along the $\mathrm{X}$ direction

$$
\phi(y, z)=\langle\phi(\boldsymbol{r})\rangle_{x}=\left\langle\frac{\rho_{A}(\boldsymbol{r})}{\rho_{A}(\boldsymbol{r})+\rho_{B}(\boldsymbol{r})}\right\rangle_{x}
$$

without loosing information about the possible lamellar orientations. From this reduced order parameter a two-dimensional structure factor can be obtained $S\left(q_{y}, q_{z}\right)=|\mathcal{F}[\phi(y, z)]|^{2}$ via a Fourier transformation. Figure 11 plots the structure factor of the intermediate state of Figure 10 separately for the stable perpendicular and the disintegrating parallel half. The perpendicular half clearly shows a dominant length scale and orientation as expected. The structure factor of disintegrating half demonstrates two separate aspects of the microemulsionlike pathway. i) the structure factor has a peaked structure indicating that the system is comprised of $\mathrm{A}$ and $\mathrm{B}$ domains with a characteristic length scale. The fact that the segregation into domains survives the transition dismisses the scenario, where the grain melts into a homogeneously mixed state of Figure 2. The dominant length scale is approximately the lamellar spacing. This finding is supported by the plot of the radial structure factor $S(|q|)$ in Figure 12 a). ii) instead of a peak, in- dicating a specific, anisotropic orientation, a ring appears. The ring indicates that all possible orientations of the lamellae are present in the system. In a small system like a simulation box this is an indication of small domains with the dominant length but no long-range orientation.
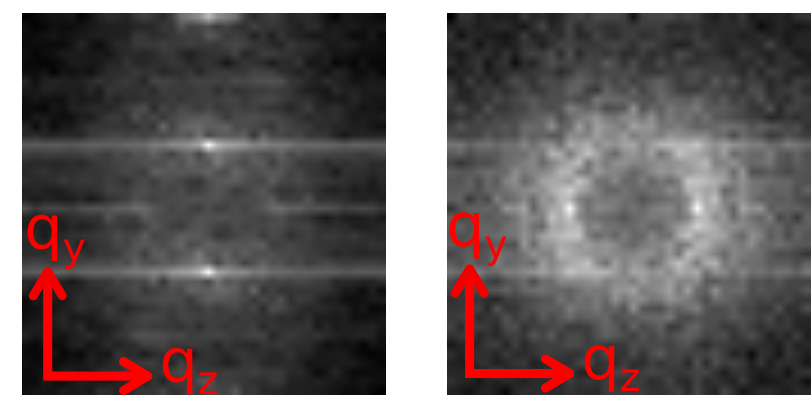

Figure 11: Two-dimensional structure factor of the sheared lamellar phase in the transition plotted logarithmically $\log \left[S\left(q_{y}, q_{z}\right)\right]$. Left: a) perpendicular left side of the simulation box. The dominant length scale and orientation is clearly visible. Right: b) disintegrating right side of the simulation box. The old dominant orientation is still dominating, but other orientations appear on the ring of the lamellar length scale.

To quantify the orientational symmetry we average the two-dimensional structure factor azimuthally

$S(\theta)=\int \mathrm{d} q_{y} \mathrm{~d} q_{z} S\left(q_{y}, q_{z}\right) \delta\left(\theta-\arcsin \left(\frac{\left|q_{y}\right|}{|q|}\right)\right)$.

For all times the two-dimensional structure factor of the left side of the simulation box exhibits two peaks, characteristic for the perpendicular orientation, see Figure $12 \mathrm{~b}$ ). $S(\theta)$ of the disintegrating, right hand side shows first the stable peak of the old orientation. But this peak becomes broader as the configuration looses its lamellar orientation until, eventually, $S(\theta)$ becomes flat at $t \approx 0.42 T_{R}$, indicating that all orientations are equally present. This intermediate state without an orientational order, but locally segregated has similarities to a microemulsion of low molecular weight surfactant, hence the name of the pathway. The indication that the peak becomes broader, flat and eventually a new peak emerges, also falsifies the rotation hypothesis (see Figure 2 a). In the latter mechanism, the col- 
lective rotation resulted in a peak that would move its position without significant broadening.
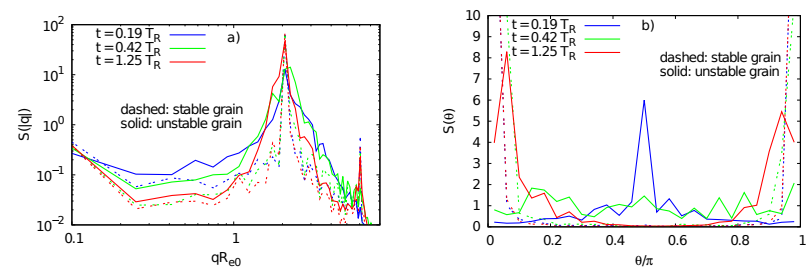

Figure 12: Structure factor $S(|q|)$ and $S(\theta)$ for three time steps of the transition process. The first time step corresponds to Figure 10 and Figure 11. The perpendicular left half of the box is indicated by dashed lines. Solid lines plot the transition of the right half. The structure factor $S(|q|)$ (left panel: a) shows the segregation at all times - the peak becomes broader, but never vanishes. The azimuthal structure factor $S(\theta)$ (right panel: b) shows that during the transition, the configuration changes from the old orientation over full anisotropy to the new orientation.

After the establishment of a flat $S(\theta)$ a peak of the new orientation emerges indicating a reorientation in the perpendicular direction. This reorientation is not perfect at first, but introduces defects. The AB-interfaces of each of these defects can be decomposed into aligned with the perpendicular orientation and aligned with the parallel parts. The parallel part is unstable in shear flow, which shows that none of the defects is stable. The defect geometry resembles that of defects, which occur in the course of structure formation from a disordered state. ${ }^{2}$ But we do not expect the defects to show the same stability or annihilation dynamics as in equilibrium or Directed Self-Assembly (DSA), because of the shear flow. Figure 13 shows that they disappear one by one with passing time. As an example Figure $13 \mathrm{~b}$ shows two opposing dislocation defects. This defect configuration is in equilibrium at $\chi N=32$ stabilized by a significant barrier against annihilation. In the presence of shear flow, however, the defect annihilation within less than three relaxation times $T_{R}$, suggesting that the process is not thermally activated, but occurs spontaneously. Finally, a defect free state with a homogeneous perpendicular orientation can be established.

To further characterize the time evolution of the transition we focus on the molecular extension
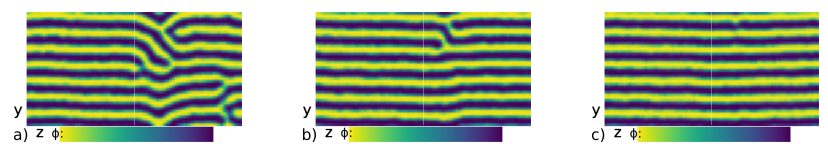

Figure 13: Spatial composition $\phi(y, z)$ for the reorientation of the lamellar direction. The first snapshot is taken after $\approx 1.2 T_{R}$ and corresponds to the latest configuration of Figure 12. The two subsequent snapshots are taken at much later times $\approx 2.7 T_{R}$ b) and $\approx 3 T_{R} \mathrm{c}$ ). They show two opposing edge dislocation defects, which annihilate each other.

$R_{\mathrm{e}}^{2}=R_{e x}^{2}+R_{e y}^{2}+R_{e z}^{2}$ as a function of time. It reveals the extension of the molecules and their orientation in the simulation box. Naturally the molecules are extended across the internal ABinterface.With the center of mass we assign each molecule to either the left or the right side of the simulation box. Thus we are able to compare the different extensions during the transition as illustrated in Figure 14.

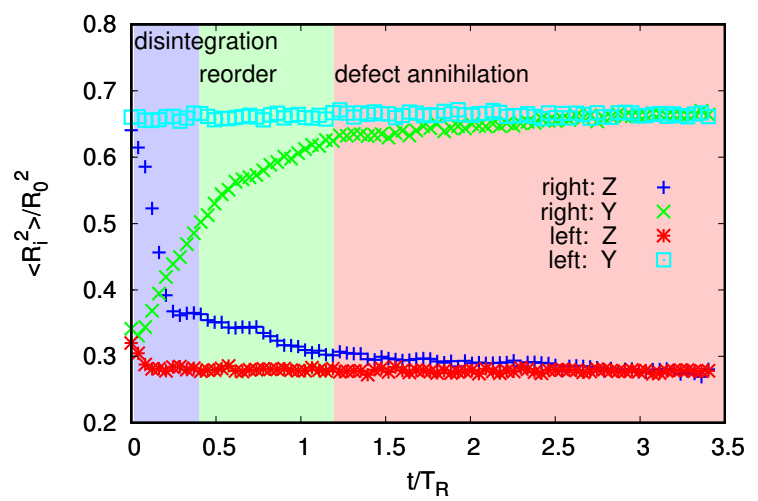

Figure 14: Molecular extension as a function of time. The background colors indicate the different stages of the microemulsion-like pathway: shear start-up (white), disintegration (blue), reorder (green) and defect annihilation (red). As a criterion for the separation of disintegration and reorder we chose the flat structure factor $S(\theta)=$ const. (Figure 12 b).

In the X-direction the molecule extension is dominated by the influence of the shear flow and no lamellar interfaces are orthogonal to this direction. So we cannot gain any insight about the transition from this quantity. Instead, we focus on the Y and $\mathrm{Z}$ component. In the initial configuration molecules are extended across the internal interfaces thus the 
two sides of the simulation box show opposite characteristics. During the transition the parallel side undergoes a transition to match its counterpart. Interestingly, the retraction in the parallel direction happens to be faster than the extension in the new perpendicular orientation. This supports the already described dynamics in two stages: first disintegration of the initial orientational order and a subsequent establishment of the orientation. Previously we described the dynamics purely via the spatial composition $\phi$. The different stages are also detectable in the single-chain extension and orientation because the chains stretch across the internal AB-interfaces. Although, the exact points in time, which separate the transition phases would be defined slightly different by the chain extension. Instead of the a flat $S(\theta)=$ const. the disintegration can be defined as time where $R_{z \|}^{2}=R_{y \|}^{2}$, refer to Figure 14. The colored time scales also highlight the different times required for the process. The shear start-up is much faster than the other processes, requiring only changes on the level of individual monomers. In addition, the disintegration of the anisotropic orientation needs significantly less time than the following establishment of the new perpendicular phase.

Falsification of the Shrinking Hypothesis During the discussion of the time evolution we already dismissed the two hypotheses of rotation and melting into a homogeneously mixed state (Figure 2 a) and c). But the hypothesis of domain shrinking has not been considered, yet. To investigate effects of the grain boundary during the transition we fold the order parameter, $\phi$ back into a single lamellar unit cell along the Y-direction.

In the disintegration stage of the transition (Figure 15) the parallel lamellar orientation vanishes homogeneously in space. In the following reorientation stage there is no registration effect of the newly forming stable lamellae at the grain boundaries. If the transition would be according to the domain-shrinking hypothesis, we would see the new lamellae forming at the grain boundary first and subsequently growing into the right side of the simulation box. This is not the case as the first lamellae dominantly form in the center of the initially misaligned grain. This is a key factor for the occurrence of defects during the transition, because

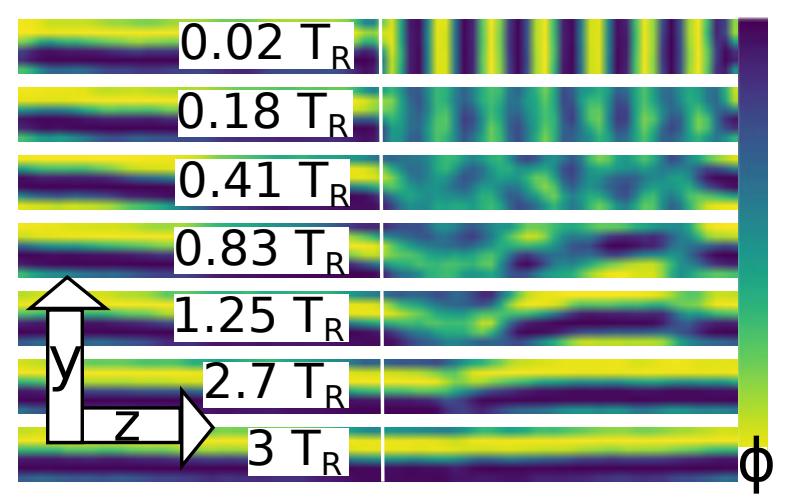

Figure 15: Time evolution of the unit cell averaged order parameter $\phi$. The newly formed lamellae do not register to the old ones at the boundary: little to no registration effect at the grain boundaries is detectable - dismissing the domain shrinking hypothesis of Figure 2 ii). The intermediate values of $\phi$ at early times do not indicate a reduction of the segregation, compare with Figure 12i). It is an artifact of the lamellar unit cell averaging, because the transition states do not have this periodicity.

the new lamellar grain is shifted with respect to the old lamellae. As the lamellae connect, defects are formed and later annihilated at the position of the initial grain boundary.

\section{Slow Transition at Lower Shear Rates in Steady Flow}

In the previous section we described the microemulsion-like pathway transition at high shear rates $\mathrm{Wi}_{\perp} \approx 200$ and $\mathrm{Wi}_{\|} \approx 94$. The transition is fast, because the influx of energy via the shear flow is sufficient to destabilize the lamellar orientation in the parallel phase immediately. We found in an earlier section that the parallel lamellae are not stable. In this section we investigate the transition for the case that the shear flux is not sufficient to disintegrate the lamellar orientation.

To study this question we investigate the exact same system as before, but we halved the induced stress compared to the microemulsion-like pathway. The resulting Weissenberg numbers in this case are: $\mathrm{Wi}_{\perp} \approx 112$ and $\mathrm{Wi}_{\|} \approx 49$. The measured Weissenberg numbers are slightly smaller than predicted from a linear relation $\mathrm{Wi} \propto j_{p}$ and can be explained by the shear-thinning characteristics of the simulated polymer melts, because of the 
non-bonded interactions. For the investigation of a the stable lamellar orientations (Figure 4) we used even lower shear rates, because a steady state was required at all simulation times. For the investigation of the transition in this section, this condition is not mandatory. A detailed investigation of the crossover shear rate, between slow transition and microemulsion-like pathway is discussed in a following section.

As in the microemulsion-like pathway, refer to Figure 9, the shear start-up is much faster than the changes in the spatial composition $\phi$. Investigating the transition with the same methods, which we used for Figure 15, we confirm that the process is described by the shrinking hypothesis of Figure 2. Figure 16 shows the time evolution of the averaged order parameter per lamellar unit cell. The boundaries move in time, shrinking the unstable parallel grain. In the remainder of this section we are discussing this mechanism in further detail.

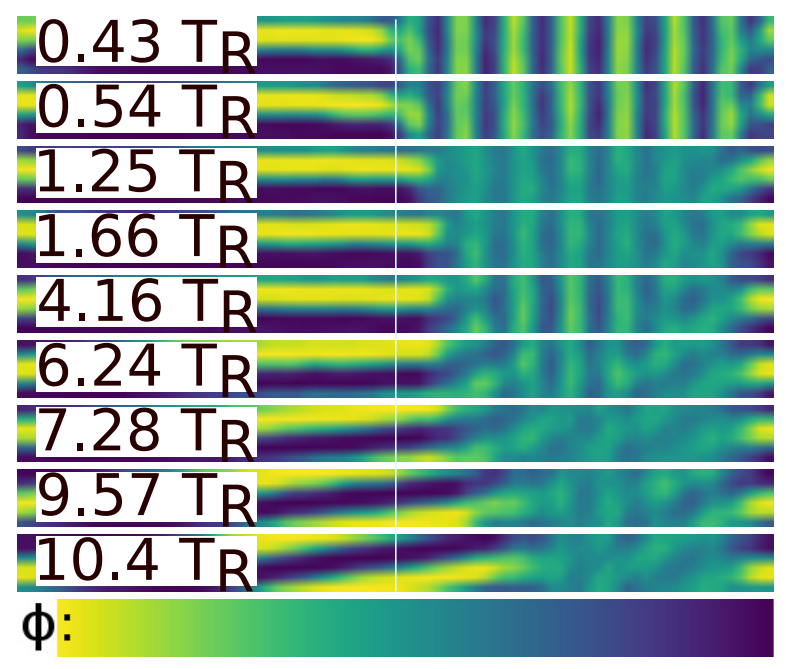

Figure 16: Over a lamellar unit cell averaged order parameter $\phi$ for the slower transition. The motion of the boundaries shrinks the unstable parallel domain. In addition, the transition requires about an order of magnitude longer than the microemulsionlike pathway. The intermediate values of $\phi$ at early times do not indicate a reduction of the microphase separation. It is an artifact of the lamellar unit cell averaging, because the transition states do not have this periodicity.

Four aspects of Figure 16 are worth to be highlighted: i) the unstable lamellae seem to be destabilized in the unstable parallel configuration. ii) the motion of the boundary is more step-wise than con- tinuous in time. iii) at later times the stable lamellae tilt significantly. iv) although, we do not establish a fully stable oriented configuration, the time required is about an order of magnitude larger than for the microemulsion-like pathway. Understanding these aspects grants a deeper insight into the mechanism.

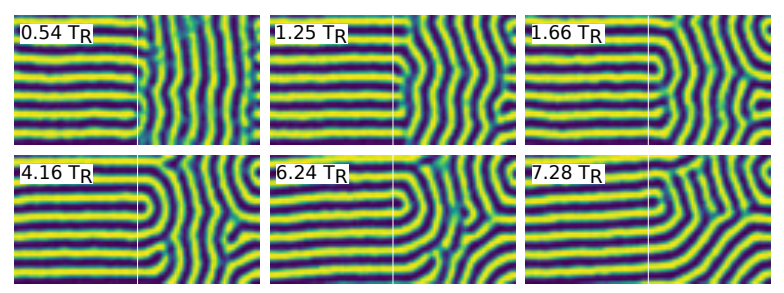

Figure 17: Two dimensional order parameter $\phi$ of the slow transition. The time evolution grants insights into the different mechanism of the transition. At later times finite size effects play an important role. Consequently, later times towards the final stable configuration are not investigated in detail.

Although, in Figure 17 the first snapshot $\left(0.54 T_{R}\right)$ shows fluctuations of parallel lamellae no homogeneous destabilization of the orientation can be detected; the lamellar order remains intact. Furthermore, the fluctuation of the lamellae are less pronounced than before; for comparison, during the microemulsion-like pathway, the unstable orientation at this time was already homogeneously disintegrated, see Figure 10. Instead, a buckling of the lamellae especially at the time between $1.25 T_{R}$ and $1.66 T_{R}$ can be observed. This buckling appears in the unit cell averaging of Figure 16 as if the lamellae would disintegrate.

A consequence of this mechanism is a merging of parallel lamellae with the perpendicular ones, wherever the parallel lamellae buckle towards a perpendicular lamellar of the same type, cf. Figure 17 at $t=1.25 T_{R}$. These merged lamellae give rise to a semi-circle shape inside the previously parallel grain, see panel corresponding to $t=4.16 T_{R}$. These semi-circular structures have an effect on both sides of the boundary. Towards the unstable grain the lamellae are compressed into the semicircular leading to a growth of the structure both in radius and into the parallel grain. This process can be observed at time $t=1.66 T_{R}$ and $t=6.24 T_{R}$. On the other side, the T-junction defects in the center of the semi-circle break-up from time to time, 
establishing the fully oriented perpendicular orientation. The explanation is straightforward: directly at a T-junction a piece of the lamellae is strongly bent and oriented in a 90 degree angle to the stable orientation. Both effects destabilize the lamella and promote a break-up of this section. Examples of such a break-up can be found at times $t=1.25 T_{R}$ and $t=7.28 T_{R}$. This sequence of break-up events is the reason for the, previously observed, stepwise growth of the stable grain.

The small size of our simulation box causes deviation from the experimental expectations as soon as the two opposing boundary structures start to interact. In experimental settings a grains is expected to contain hundreds of lamellae in contrast to the seven lamellae in the simulation box. This can be observed for times later than $6.24 T_{R}$. Because the two opposing centers of the semi-circles are not aligned, they optimize the available space by globally tilting the lamellar morphology in the entire simulation box. The tilting is especially clear in the unit-cell representation of Figure 16, but is not expected in macroscopic samples.

Another finite-size effect is the direct interaction of the outer semi-circles of the boundary. The snapshot at $t=7.28 T_{R}$ shows a break-up and merging of the outer layers. Although this is also expected in macroscopic samples, it is not of the highest importance, because it only happens at the late stages of the transition. For a much larger grain size this might affect only minor fractions of the total sample. On the other hand, the growth mechanism previously described created a defect-free perpendicular phase, but the interaction of the semi-circles might introduce regions of many, spatially concentrated defects $\left(t=7.28 T_{R}\right)$. Because this merging of two opposing semi-circles is mainly influenced by the system size, we decide to not further investigate the later stages of the grain-shrinking mechanism.

\section{Observation of the Transitions in the Labora- tory Experiments}

With the findings of the computer simulations of the previous section, we expect to observe two transition mechanisms in the experiments. In orientation experiments a fast change in the observables as the mechanic loss modulus $G^{\prime \prime}$ at the beginning, fol- lowed by a slow change can be observed. The time evolution of $G^{\prime \prime}$ for the sample PS(8.4)P2VP(8.6) in oscillatory shear experiments with $\omega_{1} / 2 \pi=1 \mathrm{~Hz}$, $\gamma_{0}=0.25$ to 2.00 for the duration of $\mathrm{t}=2700 \mathrm{~s}$ is shown in Figure 18. The change in $G^{\prime \prime}$ increases with the the shear amplitude $\gamma_{0}$. At a low value of $\gamma_{0}$ the described time evolution is clearly visible, but at higher shear amplitudes the change in $G^{\prime \prime}$ is increasingly hard to see as there are only minor changes in $G^{\prime \prime}$. The fast decrease of the loss modulus $G^{\prime \prime}$ can be associated with the microemulsionlike pathway at high shear rates. The later slow decrease is an effect of the subsequent defect annihilation. In this experiment cone-plate geometries were used, thus $\gamma$ was the same throughout the whole polymer sample.

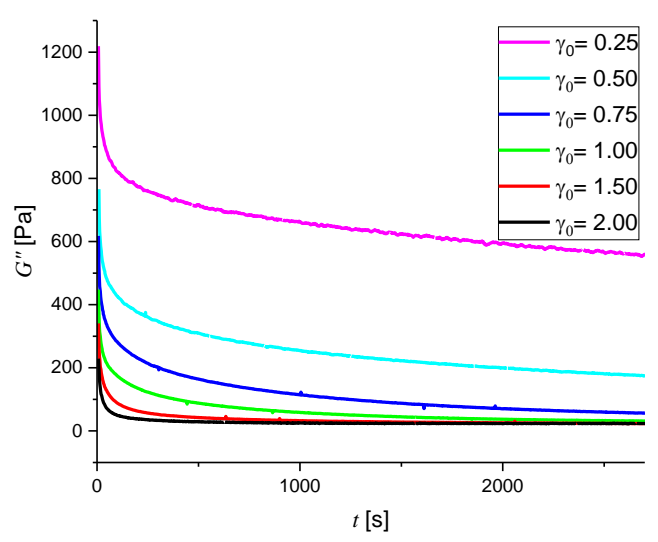

Figure 18: Time evolution of the mechanic loss modulus $G^{\prime \prime}$ in oscillatory shear experiments with $\omega_{1} / 2 \pi=1 \mathrm{~Hz}, \gamma_{0}=0.25$ to 2.00 for $t=2700 \mathrm{~s}$. A fast decrease in $G^{\prime \prime}$ can be observed at the beginning; followed by a subsequent slow process of defect annihilation.

In order to investigate this further we exploit the fact that in a parallel-plate geometry the strain varies throughout the sample. From the simulations we expect that below a certain strain rate only the slow transition occurs. In order to verify this hypothesis, we scatter X-rays at different radii of the experimentally sheared sample.

For spatially resolved scattering experiments, the diffractogram was integrated along the angle $\theta$ and the peak intensity $I$ was approximated by a Gaussian distribution. The standard deviation $\sigma$, which is used to describe the degree of orientation, showed an increase towards the center of the 
polymer disk (Figure 19). The strain rate linearly depends on the distance $R$ from the center of the rotation.

Fitting a Gaussian distribution is not optimal, because i) we do not expect $S(\theta)$ to be Gaussian distributed and indeed sizable differences can be detected and ii) as $S(\theta)$ becomes flat fitting is increasingly difficult with the diverging $\sigma$. As a solution we propose a diversity score $\lambda$ to characterize the different shapes of $S(\theta)$. The entropy $\mathbb{S}$ is a promising candidate. For a flat function, e.g. $S(\theta) \equiv 1$, the entropy becomes maximal $\mathbb{S}_{0}$. For peaked distributions the entropy decreases. We calculate the entropy

$$
\begin{gathered}
\mathbb{S}[S]=-\oint \mathrm{d} \theta A S(\theta) \log (A S(\theta)) \\
\text { with } \quad 1 / A:=\oint \mathrm{d} \theta S(\theta) \\
\text { and } \quad \mathbb{S}_{0}:=\mathbb{S}[S(\theta) \equiv 1]
\end{gathered}
$$

with the normalized structure factor $A S(\theta)$. For symmetry reasons the integral has to be evaluated only for a half of the unit circle. We empirically define the diversity score $\lambda$ such that it has the same properties as the Gaussian standard deviation $\sigma:$ i) divergence for a flat $S(\theta)$ and ii) converging to a small constant $\left(\log \left(1 / \mathbb{S}_{0}+1\right)\right)$ in the limit of a delta function.

$$
\lambda[S]=\log \left(\frac{1}{\mathbb{S}_{0}-\mathbb{S}[S]}+1\right)
$$

Both, the Gaussian width $\sigma$ and the diversity score $\lambda$, are plotted in Figure 19. They show a similar dependence on $\gamma$, so we use the well defined diversity score $\lambda$ for further interpretations, which in addition simplifies the automated analysis of noisy data obtained via simulations, compare with Figure 12.

Figure 19 clearly shows two different regions in the sample. Below a strain of about $\gamma=0.7$ the system is not completely ordered, even though the oscillatory strain has been applied for many relaxation times. In this regions the order increases from the core to a strain of about $\gamma=0.7$, which would agree with slow moving boundaries of the shrinking transition.

In contrast, the system is almost completely ordered for higher strains. Our interpretation is, that

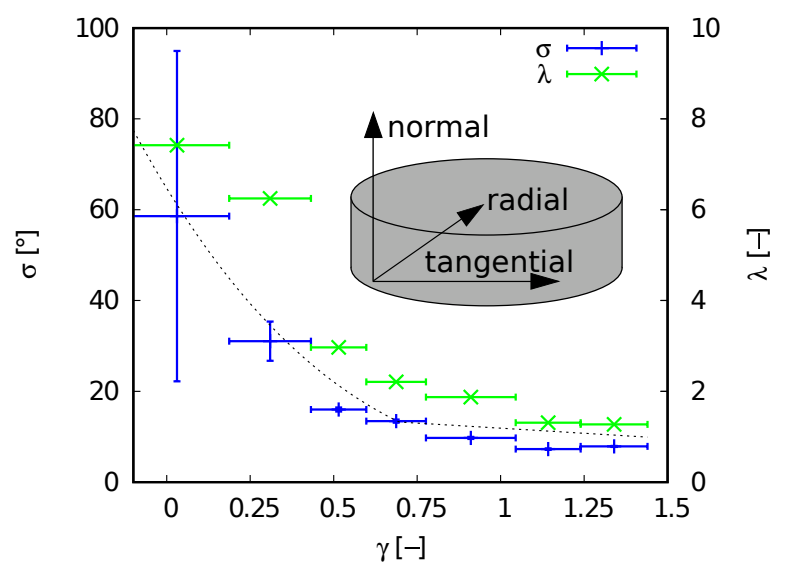

Figure 19: The degree of orientation in the normal direction is described by the standard deviation $\sigma$ of Gaussian approximations to the maxima of $I$ and the diversity score $\lambda$. Both show a similar shape and clearly demonstrate two different regions of order in the sample. A line highlighting the regime of the shrinking mechanism $\gamma \lesssim 0.7$ and the regime of the microemulsion-like pathway $\gamma \gtrsim 0.7$ is plotted to guide the eye. The inset shows the different spatial directions, in which the polymer disk of PS(8.4)P2VP(8.6) can be analyzed. As a consequence of the orientation of the lamellae, SAXS experiments were mainly conducted in the normal direction. The experimental conditions were $T=200{ }^{\circ} \mathrm{C}, \omega_{1} / 2 \pi=1 \mathrm{~Hz}, \gamma_{0}=1.5, t=3600 \mathrm{~s}$. 
in this region the microemulsion-like pathway almost immediately orders the system into the stable orientation. Only a small tendency towards a higher ordered systems can be observed at higher strains. The tendency would support the slow defect annihilation, which is the end phase of the microemulsion-like pathway.

We can use the same diversity score $\lambda[S(\theta)]$ to quantify the different transition pathways in the computer simulations. We investigated the azimuthal structure factor $S(\theta)$ already in the context of the microemulsion-like pathway (Figure $12 \mathrm{~b}$ )) and argued that the flat structure factor is an important indication of the microemulsion-like pathway, because it signals the complete loss of lamellar orientation. At this point we have to highlight difference between this structure factor obtained in experiments and computer simulations. In the experiments the X-ray beam illuminates a larger area than a single grain. Thus the resulting structure factor $S$ is an average over multiple grains. If the grains have different orientations, $S(\theta)$ can be flat; even though, each individual grain maintains a lamellar orientation. In the computer simulations we investigate with the structure factor a defined area comprised of a single grain. A flat structure factor indicates in that case the loss of lamellar orientation.

Figure 20 plots the time evolution of the diversity score $\lambda$ for the different transition pathways. The rapid increase and decrease of this score are characterizing the microemulsion-like pathways. The microemulsion like state with a flat structure factor $S(\theta)$ is indicated by the maximum of the diversity score $\lambda$. During the reorientation stage the new orientation is established and the diversity score decreases significantly. Finally, after the defect annihilation the diversity score drops to its initial low value, indicating a perfectly orientated configuration.

The characteristics of the slow transition are different. As the grain boundary moves into the unstable grain, the proportions of the lamellar orientations gradually change. As a result the diversity score of the structure factor $S(\theta)$ slowly increases. The simulation time is not sufficient for the diversity score to decrease again, indicating a completely orientated grain. The maximum of is still lower than for the microemulsion-like pathway, because

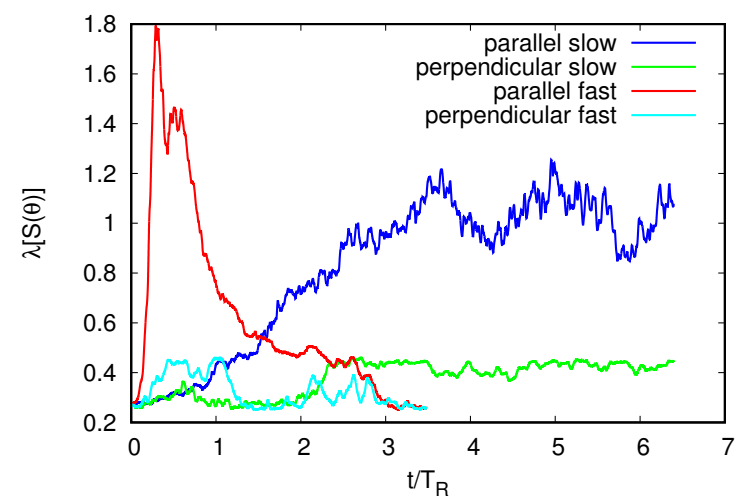

Figure 20: Time evolution of the diversity score $\lambda[S(\theta)]$ (Equation 11) of the azimuthal structure factor. For the microemulsion-like pathway $\lambda$ rises to a maximum, at which $S(\theta)$ is flat, afterwards the score decreases in the reorientation and defectannihilation stage. As a complete stable orientation is reached the score drops to its initial value. The slow transition does not exhibit this intermediate stage - instead the score slowly and monotonously increases over time. The shape of the two curves easily separates the two transition types. The score of the two stable halves show similar features of a low score $\lambda$, because the structure does not change.

two lamellar orientations (parallel and perpendicular) coexist during the transition, in contrast to the microemulsion like state where all possible orientations are present.

The stable half of simulation box shows for both cases a similar level of the diversity score, which is expected since the configuration is stable and therefore dominated by the stable lamellar orientation at all times.

\section{Crossover Shear Rate Separating the Transi- tion Pathways}

Two different transition mechanisms separated by the shear rate have been discussed so far. In this section we determine the applied constant stress that separates the two transitions with computer simulations.

The slow transition, shrinking of the unstable grain, depends on the grain boundary to the stable grain. We use an initial configuration similar to the one we used before, Figure 8 . The only difference is that we fill the box completely with the 
unstable parallel lamellar orientation. Without the grain boundary this orientation is metastable for shear rates corresponding to the slow transition, but unstable for the shear rates corresponding to the microemulsion-like pathway.

We perform a binary search to find the separation between the two transitions mechanisms. The simulation time of each configuration is $2 T_{R}$ relaxation times. This time is longer than required for the intermediate, microemulsion like state of the fast transition $t \approx 0.42 T_{R}$, but still not long enough to exhibits a full slow transition driven by nucleation and growth. Because of the absence of a grain boundary a nucleus with the stable perpendicular orientation is required, before a stable phase can be formed. We have two options to determine the transition type. i) the time evolution of the diversity score $\lambda$, compare with Figure 20 and ii) the spatial composition of the final state. If the final state is still in the parallel configuration, the shear rate corresponds to the slow transition, otherwise to the unstable fast transition mechanics.

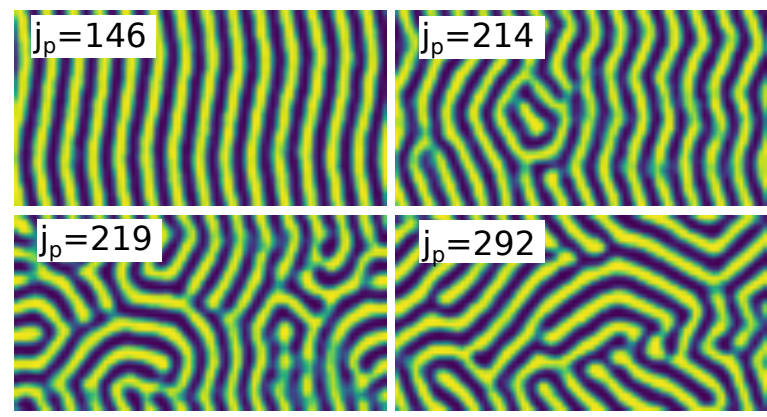

Figure 21: Final configurations after a simulation time of $2 T_{R}$ at different shear rates. It is clear to distinguish the parallel (slow) from the multioriented (fast) configurations. The unit of the stress are in reduced units of the simulation.

Figure 21 shows the final configurations of different shear rates. The highest applied shear stress, which still results in the parallel orientation is $j_{p} R_{e o}^{3} / k_{B} T \approx 214$ in reduced simulation units. The next higher shear stress of $j_{p} R_{e o}^{3} / k_{B} T \approx 219$ does not show a clear dominant orientation, indicating an ongoing microemulsion-like pathway. The shape of the diversity scores $\lambda$ as a function of time strengthens this analysis. All curves above $j_{p} R_{e o}^{3} / k_{B} T \approx 219$ show an increase to a higher maximum, indicating the microemulsion like intermediate state. The simulation time is too short for the configurations to completely re-orient in the new stable orientation, thus the diversity score does not decrease again, see Figure 22. We expect to find the occurrence of this first maximum at later times as the shear stress $j_{p}$ decreases. Finally, this time diverges at the crossover shear rate, separating both mechanisms.

In contrast at lower shear stresses the diversity score increases only slowly and not as high as for the microemulsion-like pathway. This increase can be explained by the already discussed buckling of the lamellae and small defects in the lamellae configuration. We expect these defects to be part of the transition mechanism out of the metastable state towards the globally stable perpendicular state.

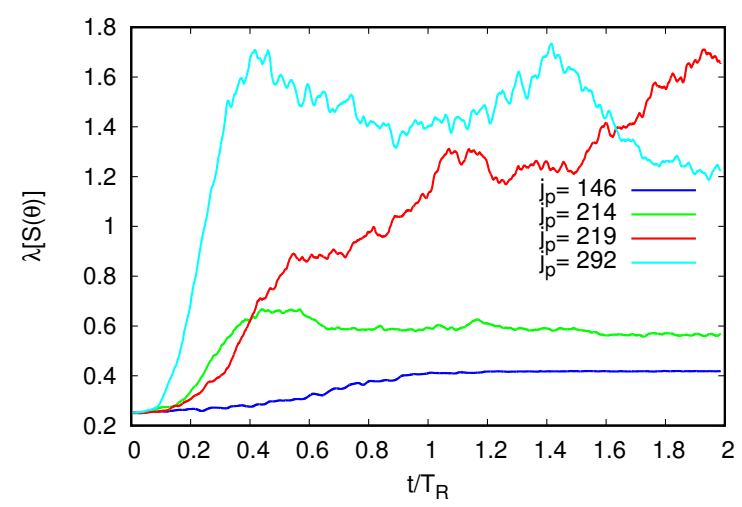

Figure 22: Diversity score $\lambda$ as a function of time. The configurations following the microemulsionlike pathway increase to a high maximum, while the slow transitions show a gradual increase with a lower maximum plateau value.

Combining both tools we can conclude that the crossover constant stress is at $j_{p} R_{e o}^{3} / k_{B} T=216 \pm$ 3 , which corresponds to a Weissenberg number of $\mathrm{Wi}_{\text {crit. }}=70.5 \pm 0.3$ in the perpendicular orientation. At this shear we cannot differentiate between a microemulsion like state of the fast transition and a slow transition with many nucleation centers. This can be seen analogous to th see crossover for the spinodal decomposition in equilibrium statistical mechanics

\section{Transitions in Oscillatory Shear}

The experiments of this study were conducted with oscillatory shear experiments, whereas the simulation results were obtained in constant shear situa- 
tions. In this section we investigate the effect of oscillatory shear on our predictions of the transitions. For this setup we use the RNEMDS with a sinusoidal stress input $J_{\text {target }}$. The maximum stress of this input is chosen such that a microemulsionlike pathway is expected. We vary the frequency $f$ of this input and study the resulting dynamics. As the stress propagation in the system is not instantaneous but fast, compare with Figure 9, we can assume no interference between the stress propagation and the oscillatory shear for all selected frequencies.

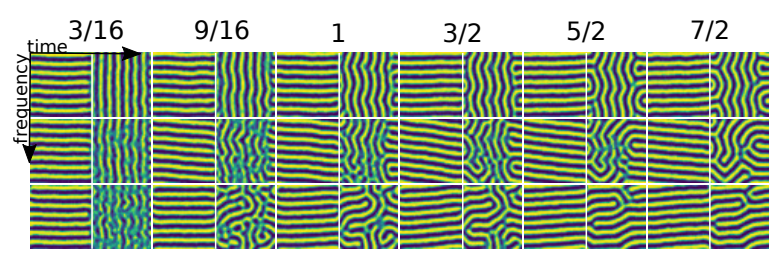

Figure 23: Time evolution of the configurations at different frequencies. The top row is at an oscillation frequency $f T_{R}=4$, the middle row at $f T_{R}=1$ and the final row at $f T_{R}=1 / 4$. The numbers at the top indicate the time in units of the relaxation time $T_{R}$. Even though the maximum shear rate promotes a disintegration of the unstable lamellar orientation, this can only be observed for the lowest frequency $f T_{R}=1 / 4$. Higher frequencies have similarities with the slow shrinking transition.

Figure 23 shows the time evolution of the compositions for three different frequencies. For the highest frequency $f T_{R}=4$ the expected disintegration of the lamellar orientation cannot be observed. Instead we see a buckling of the lamellae followed by the formation of semi-circles and a propagation of these into the unstable phase. Even though, the maximum frequency would allow a disintegration of the lamellar orientation, we observe the slower grain-shrinking mechanism. Only at lower frequencies $f T_{R}=1$ we can see a disintegration of the lamellar orientation, but even here the process is not fully established. Instead at $t=T_{R}$ the unstable orientation is more prominent than at $t=9 / 16 T_{R}$. Despite this beginning disintegration, the dynamics is still close to the slow shrinking transition. At the lowest frequency we considered $f T_{R}=1 / 4$, we see the expected dynamics of the fast disintegration dynamics, followed by a reorientation and defect annihilation. In the example of Figure 23 the defects dislocations. The annihilation of this defect is expected to be long, because it has to propagate through half the system size. Therefore, we did not simulate until a fully ordered system is observed.

Our interpretation is, that the oscillation period of high stress is not long enough to fully disintegrate the lamellar orientation. Instead the disintegration begins, but halfway through the stress is not sufficient and the unstable orientation can reform. For intermediate frequencies this partial disintegration promotes the formation of stable orientation at the grain boundary, but it is still the shrinking mechanism. Consequently, the ordering time still scales with the grain size.

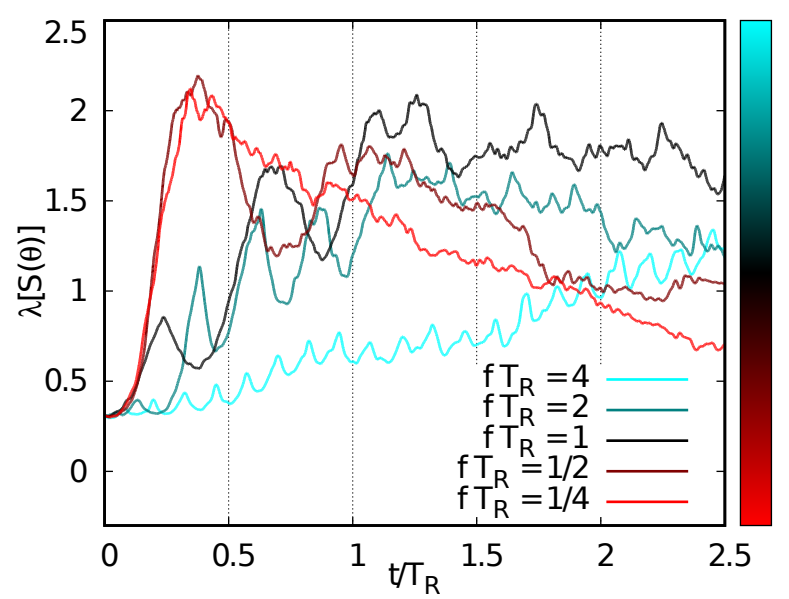

Figure 24: Diversity score $\lambda$ for the oscillatory shear simulation of the ordering process. The score is calculated from the structure factor of only the simulation half of the unstable orientation. At the lowest frequency we see the expectations of the microemulsion-like pathway. At higher frequencies the increase of $\lambda$ is significantly slowed down and moreover oscillating with the input frequency.

To investigate further our interpretation we can examine the azimuthal structure factor $S(\theta)$ via the introduced diversity score $\lambda$, see Equation 11 . Figure 24 shows the time evolution of this diversity score for five different frequencies. For the lowerst $f T_{R}=1 / 4$ we see the expected shape of the microemulsion-like pathway: a rapid increase of $\lambda$ to a maximum, indicating the disintegration of the orientation with a flat $S(\theta)$ followed by a slow decrease of $\lambda$, which corresponds to reorientation in the new stable direction establishing a single peaked $S(\theta)$. 
Increasing the frequency inhibits this dynamics. At first $\lambda$ increases as fast as at the lowest frequency, but with reversing shear direction the diversity score $\lambda$ decreases again. This is consistent with our interpretation that the disintegration cannot be fully established in a single oscillation period. For the highest frequency this is dramatic: At each iteration almost the entire disintegration can be reversed - leading to no flat $S(\theta)$ at all. Instead $\lambda$ oscillates with the input frequency and slowly increases with the progression of the slow shrinking of the unstable grain.

We conclude that oscillatory shear can slow down the transition from the unstable to the stable lamellar orientation. This also explains, why the orientation of the sample requires more relaxation times than predicted by our simulations. For high frequencies also the kind of transition can be changed from the fast microemulsion-like pathway to the slow shrinking transition.

Molecular Orientation In the computer simulations we have directly access to the molecule orientation - enabling an insight in the chain dynamics during this transitions.
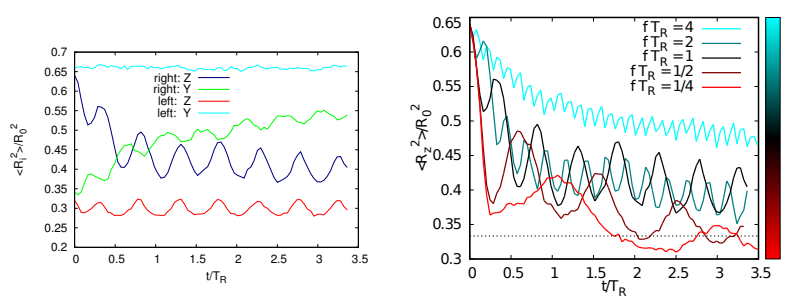

Figure 25: Time evolution of the components of the average end-to-end distance perpendicular to the shear direction. Left: i) plots all direction both in the stable and unstable half of the simulation box for the frequency $f T_{R}=1$. Right: ii) varies the frequency, but plots only the $z$ component $\left\langle R_{e z}^{2}\right\rangle$ for the unstable half.

Figure 25 i) shows the molecule orientation similar to Figure 14. The extension of the molecules oscillate in phase with the input frequency ${ }^{2}$, only in the stable simulation half the direction perpendicular to the boundaries $\left\langle R_{e y}^{2}\right\rangle$ is inhibited to oscillate. This is intuitive, since the interfaces restrict

\footnotetext{
${ }^{2}$ The frequency appears to be doubled to the input, because we measure the squared end-to-end distance $R_{\mathrm{e}}^{2}$. So we see the response to the input squared.
}

the motion of the molecules. More surprising is the oscillation of the $z$ component of the end-toend vector in the unstable half, since this is also perpendicular to the interfaces. But indeed this is consistent with our previous findings: the cyclic motion of the polymers in the unstable phase destabilize the phase - similar to polymer brushes. The amplitude of this cyclic motion scales with the input stress. As this motion destabilizes the interface in the microemulsion-like pathway, an oscillation in the amplitude controls the overall disintegration of the lamellar orientation.

As the motion $\left\langle R_{e z}^{2}\right\rangle$ drives the systems along the microemulsion-like pathway we examine it in more detail for different frequencies in Figure 25 ii). In all cases we observe an oscillation with the input frequency as expected. But the amplitude of the molecule oscillation strongly depends on the frequency. For high frequencies the amplitude is significantly smaller, since the chains are subjected to the shear stress for a shorter time period, before they can relax their stretching if this amplitude is not large enough to initiate the new stable orientation. Instead at high frequencies we observe a general trend of the oscillating curve towards the expected value on a longer time scale. We expect this drift to stem from the slow shrinking transition. For low frequencies the amplitude enclose the equilibrium values of the other transition and therefore drives the fast transition along the microemulsionlike pathway. After the disintegration, we see an oscillation around the equilibrium similar to the stable simulation half, compare with Figure 25 (i).

Our observation of the molecule orientation in oscillatory shear driven transition encourages our interpretation, that the cyclic motion of the polymers at the brush interfaces drives the fast disintegration of the unstable orientation.

\section{Conclusion and Outlook}

The stability of lamellar orientations in shear flow has been investigated by large-scale simulation of a soft, highly coarse-grained particle model and experiment. In accordance with previous simulation results, ${ }^{6,21,22}$ we find the perpendicular configuration to be stable for all investigated shear rates. Although, previous results were obtained by different 
models, we observe the same physics and quantify the non-equilibrium steady state by the Rayleighian. These findings of the computer simulations have been confirmed by our experiments.

Then we investigated the reorientation of a grain with the unstable orientation embedded in a matrix of lamellae with the stable, perpendicular orientation. We observe a microemulsion-like pathway from the parallel orientation to the stable orientation at high Weissenberg numbers $\mathrm{Wi}=94-200$, which we assume to be close to the experimental Large Amplitude Oscillatory Shear (LAOS) experiments. Three suggestions about the mechanism of the transition have been made: The rotation hypothesis has been falsified because no collective motion of the lamellae could be measured, and the orientational order of the lamellar phase is destroyed in the first stage. During the transition, the system maintains its segregation into A-rich and B-rich domains with an unchanged characteristic length scale. This disqualifies the melting hypothesis, which predicts a homogeneous composition as the intermediate state. We also rule out the last hypothesis of domain shrinking by studying the boundary between two grains. No registration of the lamellae or shrinking/growing of the grains at their boundaries is observed.

Instead the transition can be described as a disintegration of the lamellar orientation. The transition region undergoes an intermediate state of complete lamellar isotropy followed by a reorganization into the new stable lamellar orientation. Figure 26 sketches this transition for comparison with the proposed hypotheses in Figure 2. At the shear rate of the microemulsion-like pathway the parallel lamellar orientation is unstable, consequently the transition can be seen as an analogue of a spinodal phase transformation.

The second mechanism that we investigated occurs at lower shear rates of $\mathrm{Wi}=49-112$. It can be best described by a growing and shrinking process of the stable and unstable grains, respectively. At the grain boundary, semi-circle structures of the parallel grain are formed. The semi-circle grows with time in radius and propagates into the unstable grain. This process produces an almost defectfree perpendicular phase. Only at late stages, as opposing semi-circle structures start to interact, localized defects are formed. In contrast to the

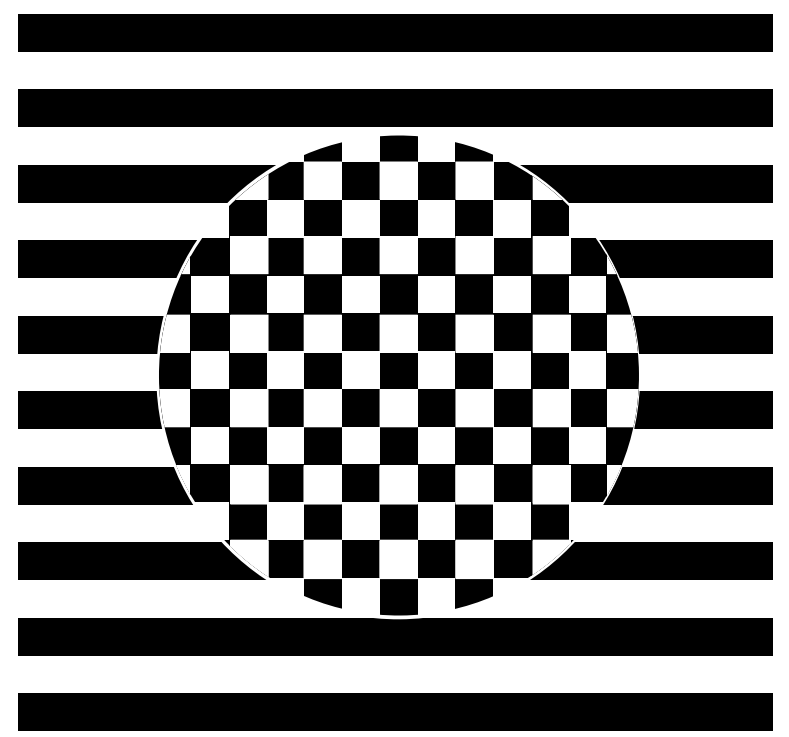

Figure 26: Schematic sketch of the transition of an unstable grain in a stable matrix. The unstable grain passes through a completely isotropic, but segregated state. Even though, the sketch approximates the transition for an intuition.

microemulsion-like pathway, the parallel lamellar orientation appears to be metastable.

In comparison with our experimental studies of the same system, we could find indications for the same two transition in the samples. For low strain rates, the ordering of the sample takes significantly longer than for higher strain rates. This coincides with our findings about the two types of transitions. In further experimental studies we aim to verify the two different transition via the defect distributions with TEM real space images of the sample. Because defects are relatively stable it is likely that defects can be observed and analyzed after shear experiments ordering the sample. If regions of homogeneously distributed defects are observed, the mechanism of ordering was likely the along the microemulsion-like pathway because defects are homogeneously formed after the disintegration of the lamellar orientation. On the other hand, if localized lines of high defect densities are observed, they may originate from the fusion of semi-circle structures - a signature of the growing and shrinking mechanism. In addition, if a sample is not fully ordered with the growing and shrinking mechanism, we expected that buckled lamellae and semi-circle structures can be detected.

In order to compare better to experiments we 
have also investigated oscillatory shear situation via computer simulations. We found that high frequencies slow down the transition in general. For high frequencies even the type of the transition can be changed from the fast, spinodal-like, to the slow growing and shrinking transition. The comparison of the diversity score $\lambda[S(\theta)]$ of experiment and simulation indicate that our experiments are conducted at frequencies, which slow down the transition to the growing and shrinking mechanism.

\section{Acknowledgements}

We thank the German Science Foundation for financial support (DFG Mu 1674/16-1; DFG WI 1911/22-1). The authors gratefully acknowledge the computing time granted by the John von Neumann Institute for Computing (NIC) and provided on the supercomputer JURECA ${ }^{63}$ at Jülich Supercomputing Centre (JSC). We also gratefully acknowledge the computing time from the HLRN Hannover and the GWDG Göttingen. The authors thank DESY, Hamburg, for access to the X-ray beam and Dr. E. Stellamanns and Dr. M. Sprung for their help at beamline P10.

\section{References}

(1) Matsen, M. W. The standard Gaussian model for block copolymer melts. Journal of Physics: Condensed Matter 2001, 14, R21.

(2) Li, W.; Müller, M. Defects in the selfassembly of block copolymers and their relevance for directed self-assembly. Annual Review of Chemical and Biomolecular Engineering 2015, 6, 187-216.

(3) Matsen, M. W.; Bates, F. S. Unifying Weakand Strong-Segregation Block Copolymer Theories. Macromolecules 1996, 29, 10911098.

(4) Bates, F. S.; Fredrickson, G. H. Block Copolymer Thermodynamics: Theory and Experiment. Annual Review of Physical Chemistry 1990, 41, 525-557.
(5) Meins, T.; Hyun, K.; Dingenouts, N.; Fotouhi Ardakani, M.; Struth, B.; Wilhelm, M. New Insight to the Mechanism of the ShearInduced Macroscopic Alignment of Diblock Copolymer Melts by a Unique and Newly Developed Rheo-SAXS Combination. Macromolecules 2012, 45, 455-472.

(6) Peters, B. L.; Ramírez-Hernández, A.; Pike, D. Q.; Müller, M.; de Pablo, J. J. Nonequilibrium Simulations of Lamellae Forming Block Copolymers under Steady Shear: A Comparison of Dissipative Particle Dynamics and Brownian Dynamics. Macromolecules 2012, 45, 8109-8116.

(7) Rottler, J.; Srolovitz, D. J. Mechanism of Shear-Induced Alignment in Bilayer Thin Films of Spherical Particles. Physical Review Letters 2007, 98, 175503.

(8) Marencic, A. P.; Wu, M. W.; Register, R. A.; Chaikin, P. M. Orientational order in sphereforming block copolymer thin films aligned under shear. Macromolecules 2007, 40, 7299_ 7305 .

(9) Wu, M. W.; Register, R. A.; Chaikin, P. M. Shear alignment of sphere-morphology block copolymer thin films with viscous fluid flow. Physical Review E 2006, 74, 040801.

(10) Arya, G.; Rottler, J.; Panagiotopoulos, A. Z.; Srolovitz, D. J.; Chaikin, P. M. Shear Ordering in Thin Films of Spherical Block Copolymer. Langmuir 2005, 21, 11518-11527, PMID: 16285835.

(11) Luo, K.; Yang, Y. Orientational phase transitions in the hexagonal cylinder phase and kinetic pathways of lamellar phase to hexagonal phase transition of asymmetric diblock copolymers under steady shear flow. Polymer 2004, 45, 6745-6751.

(12) Angelescu, D. E.; Waller, J. H.; Adamson, D. H.; Deshpande, P.; Chou, S. Y.; Register, R. A.; Chaikin, P. M. Macroscopic Orientation of Block Copolymer Cylinders in Single-Layer Films by Shearing. Advanced Materials 2004, 16, 1736-1740. 
(13) Ren, S.; Hamley, I.; Teixeira, P.; Olmsted, P. Cell dynamics simulations of shear-induced alignment and defect annihilation in stripe patterns formed by block copolymers. Physical Review E 2001, 63, 041503.

(14) Langela, M.; Wiesner, U.; Spiess, H. W.; Wilhelm, M. Microphase Reorientation in Block Copolymer Melts As Detected via FT Rheology and 2D SAXS. Macromolecules 2002, 35, 3198-3204.

(15) Struth, B.; Hyun, K.; Kats, E.; Meins, T.; Walther, M.; Wilhelm, M.; Grübel, G. Observation of New States of Liquid Crystal 8CB under Nonlinear Shear Conditions as Observed via a Novel and Unique Rheology/Small-Angle X-ray Scattering Combination. Langmuir 2011, 27, 28802887.

(16) Koppi, K. A.; Tirrell, M.; Bates, F. S.; Almdal, K.; Colby, R. H. Lamellae orientation in dynamically sheared diblock copolymer melts. Journal de Physique II 1992, 2, 19411959.

(17) Patel, S.; Larson, R.; Winey, K.; Watanabe, H. Shear orientation and rheology of a lamellar polystyrene-polyisoprene block copolymer. Macromolecules 1995, 28, 4313-4318.

(18) Riise, B. L.; Fredrickson, G. H.; Larson, R. G.; Pearson, D. S. Rheology and shearinduced alignment of lamellar diblock and triblock copolymers. Macromolecules 1995, 28, 7653-7659.

(19) Chen, Z.-R.; Kornfield, J. A. Flow-induced alignment of lamellar block copolymer melts. Polymer 1998, 39, 4679-4699.

(20) Pryamitsyn, V.; Ganesan, V. Correlations in block copolymers under shear. Macromolecules 2002, 35, 9847-9850.

(21) Fraser, B.; Denniston, C.; Müser, M. H. On the orientation of lamellar block copolymer phases under shear. The Journal of Chemical Physics 2006, 124, 104902.
(22) Liu, W.; Qian, H.-J.; Lu, Z.-Y.; Li, Z.S.; Sun, C.-C. Dissipative particle dynamics study on the morphology changes of diblock copolymer lamellar microdomains due to steady shear. Physical Review E 2006, 74, 021802.

(23) Fraser, B.; Denniston, C.; Müser, M. H. Diffusion, elasticity, and shear flow in selfassembled block copolymers: A molecular dynamics study. Journal of Polymer Science Part B: Polymer Physics 2005, 43, 970-982.

(24) Shagolsem, L.; Kreer, T.; Sommer, J.-U. Shear-Induced Ordering in Thin Films of Diblock Copolymer Melts. ACS Macro Letters 2014, 3, 1201-1204.

(25) Shagolsem, L. S.; Kreer, T.; Galuschko, A.; Sommer, J.-U. Diblock-copolymer thin films under shear. The Journal of Chemical Physics 2016, 145, 164908.

(26) Guo, H.; Kremer, K.; Soddemann, T. Nonequilibrium molecular dynamics simulation of shear-induced alignment of amphiphilic model systems. Physical Review E 2002, 66, 061503.

(27) Soddemann, T.; Auernhammer, G. K.; Guo, H.; Dünweg, B.; Kremer, K. Shearinduced undulation of smectic-A: Molecular dynamics simulations vs. analytical theory. The European Physical Journal E 2004, 13, 141-151.

(28) Kodama, H.; Doi, M. Shear-Induced Instability of the Lamellar Phase of a Block Copolymer. Macromolecules 1996, 29, 2652-2658.

(29) Heck, M.; Schneider, L.; Müller, M.; Wilhelm, M. Diblock Copolymers with Similar Glass Transition Temperatures in Both Blocks for Comparing Shear Orientation Processes with DPD Computer Simulations. Macromolecular Chemistry and Physics 2018,

(30) Müller, M. Studying Amphiphilic Selfassembly with Soft Coarse-Grained Models. Journal of Statistical Physics 2011, 145, 9671016. 
(31) Groot, R. D.; Warren, P. B. Dissipative Particle Dynamics: Bridging the Gap Between Atomistic and Mesoscopic Simulation. The Journal of Chemical Physics 1997, 107, 44234435.

(32) Groot, R. D.; Warren, P. B. Dissipative particle dynamics: Bridging the gap between atomistic and mesoscopic simulation. Journal of Chemical Physics 1997, 107, 4423.

(33) Phillips, C. L.; Anderson, J. A.; Glotzer, S. C. Pseudo-Random Number Generation for Brownian Dynamics and Dissipative Particle Dynamics Simulations on GPU Devices. Journal of Computational Physics 2011, 230, 7191-7201.

(34) Dünweg, B.; Paul, W. Brownian dynamics simulations without Gaussian random numbers. International Journal of Modern Physics C 1991, 2, 817-827.

(35) Müller-Plathe, F. Reversing the Perturbation in Nonequilibrium Molecular Dynamics: An Easy Way to Calculate the Shear Viscosity of Fluids. Physical Review E 1999, 59, 48944898.

(36) HOOMD-blue user documentation. https: //hoomd-blue.readthedocs.io/ en/stable/, 2018; Accessed: 2018-2405 .

(37) Anderson, J. A.; Lorenz, C. D.; Travesset, A. General Purpose Molecular Dynamics Simulations Fully Implemented on Graphics Processing Units. Journal of Computational Physics 2008, 227, 5342 - 5359.

(38) Phillips, C. L.; Anderson, J. A.; Glotzer, S. C. Pseudo-random number generation for Brownian Dynamics and Dissipative Particle Dynamics simulations on GPU devices. Journal of Computational Physics 2011, 230, 71917201.

(39) HOOMD-blue Bitbucket project. https: / / bitbucket.org/glotzer/ hoomd-blue, 2017; Accessed: 2017-1708.
(40) Glaser, J.; Nguyen, T. D.; Anderson, J. A.; Lui, P.; Spiga, F.; Millan, J. A.; Morse, D. C.; Glotzer, S. C. Strong scaling of generalpurpose molecular dynamics simulations on GPUs. Computer Physics Communications 2015, 192, 97-107.

(41) Lynd, N. A.; Meuler, A. J.; Hillmyer, M. A. Polydispersity and block copolymer selfassembly. Progress in Polymer Science 2008, 33, 875-893.

(42) Braun, D. Polymer synthesis: theory and practice: fundamentals, methods, experiments, 4th ed.; Springer: Berlin/New York, 2005.

(43) Hadjichristidis, N.; Pitsikalis, M.; Iatrou, H. In Block Copolymers I; Abetz, V., Ed.; Springer: Berlin/Heidelberg, 2005; Vol. 189.

(44) Matyjaszewski, K.; Müller, A. H. Controlled and living polymerizations: from mechanisms to applications; John Wiley \& Sons, 2009.

(45) Ekenstein, A. v.; Meyboom, R.; ten Brinke, G.; Ikkala, O. Determination of the Flory-Huggins Interaction Parameter of Styrene and 4-Vinylpyridine Using Copolymer Blends of Poly(styrene-co4-vinylpyridine) and Polystyrene. Macromolecules 2000, 33, 3752-3756.

(46) Shull, K. R.; Kramer, E. J.; Hadziioannou, G.; Tang, W. Segregation of block copolymers to interfaces between immiscible homopolymers. Macromolecules 1990, 23, 4780-4787.

(47) Clarke, C. J.; Eisenberg, A.; La Scala, J.; Rafailovich, M. H.; Sokolov, J.; Li, Z.; Qu, S.; Nguyen, D.; Schwarz, S. A.; Strzhemechny, Y.; Sauer, B. B. Measurements of the Flory-Huggins Interaction Parameter for Polystyrene-Poly(4-vinylpyridine) Blends. Macromolecules 1997, 30, 4184-4188.

(48) Leibler, L. Theory of Microphase Separation in Block Copolymers. Macromolecules 1980, 13, 1602-1617.

(49) Doi, M.; Edwards, S. The Theory of Polymer Dynamics; Clarendon Press: New York, 1988. 
(50) Meins, T.; Hyun, K.; Dingenouts, N.; Fotouhi Ardakani, M.; Struth, B.; Wilhelm, M. New Insight to the Mechanism of the ShearInduced Macroscopic Alignment of Diblock Copolymer Melts by a Unique and Newly Developed Rheo-SAXS Combination. Macromolecules 2012, 45, 455-472.

(51) Onsager, L. Reciprocal relations in irreversible processes. II. Physical Review 1931, $38,2265$.

(52) Doi, M. Onsager's variational principle in soft matter. Journal of Physics: Condensed Matter 2011, 23, 284118.

(53) Koopman, E. A.; Lowe, C. P. Advantages of a Lowe-Andersen Thermostat in Molecular Dynamics Simulations. The Journal of Chemical Physics 2006, 124, 204103.

(54) Landau, L. D.; Lifschitz, E. M. Lehrbuch Theoretischer Physik; Akademischer Verlag Berlin, 1966; Vol. 6.

(55) Müller, M.; Pastorino, C. Cyclic motion and inversion of surface flow direction in a dense polymer brush under shear. Europhysics Letters 2007, 81, 28002.

(56) Pastorino, C.; Müller, M. Mixed brush of chemically and physically adsorbed polymers under shear: Inverse transport of the physisorbed species. Journal of Chemical Physics 2014, 140, 014901.

(57) Meins, T.; Dingenouts, N.; Kübel, J.; Wilhelm, M. In Situ Rheodielectric, ex Situ 2DSAXS, and Fourier Transform Rheology Investigations of the Shear-Induced Alignment of Poly(styrene- $b$-1,4-isoprene) Diblock Copolymer Melts. Macromolecules 2012, 45, 7206-7219.

(58) Winey, K. I.; Patel, S. S.; Larson, R. G.; Watanabe, H. Interdependence of shear deformations and block copolymer morphology. Macromolecules 1993, 26, 2542-2549.

(59) Winey, K. I.; Patel, S. S.; Larson, R. G.; Watanabe, H. Morphology of a lamellar diblock copolymer aligned perpendicular to the sample plane: transmission electron microscopy and small-angle X-ray scattering. Macromolecules 1993, 26, 4373-4375.

(60) Stukowski, A. Visualization and analysis of atomistic simulation data with OVITO-the Open Visualization Tool. Modelling and Simulation in Materials Science and Engineering 2009, 18, 015012.

(61) Breiner, U.; Krappe, U.; Thomas, E. L.; Stadler, R. Structural Characterization of the "Knitting Pattern" in Polystyreneblock -poly(ethylene- co-butylene)- blockpoly(methyl methacrylate) Triblock Copolymers. Macromolecules 1998, 31, 135-141.

(62) Sandia National Labs and Kitware Inc and Los Alamos National Labs, ParaView. 20002017; http://www.paraview.org.

(63) Krause, D.; Thörnig, P. JURECA: Generalpurpose supercomputer at Jülich Supercomputing Centre. Journal of large-scale research facilities 2016, 2, 62. 


\section{Graphical TOC Entry}

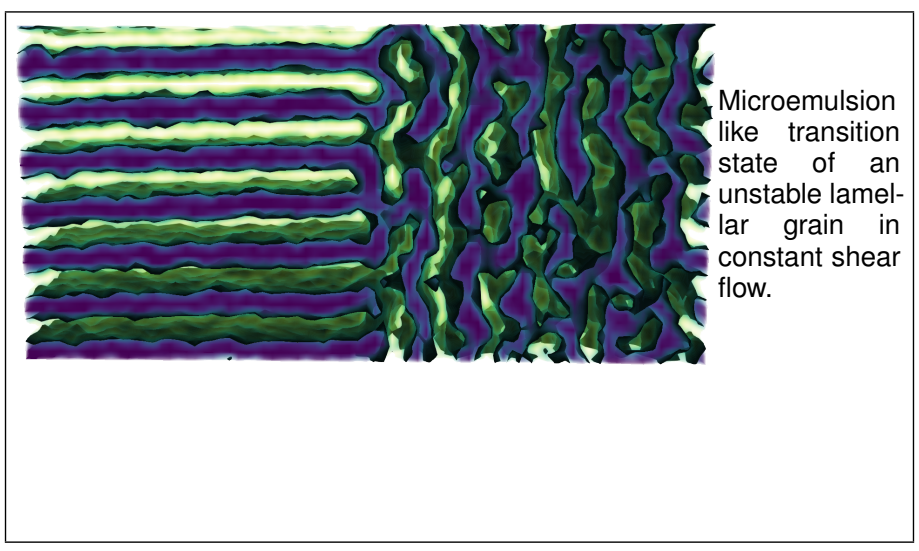

